\title{
Completeness of the isomorphism problem for separable $\mathrm{C}^{*}$-algebras
}

\author{
Marcin Sabok ${ }^{1,2}$
}

Received: 20 November 2014 / Accepted: 26 August 2015 /

Published online: 24 September 2015

(C) The Author(s) 2015. This article is published with open access at Springerlink.com

\begin{abstract}
This paper studies the descriptive set-theoretical complexity of the isomorphism problem for separable $\mathrm{C}^{*}$-algebras. We prove that the isomorphism problem for separable (simple, $\mathrm{AI}$ ) $\mathrm{C}^{*}$-algebras is complete in the class of orbit equivalence relations. This means that any isomorphism problem arising from a continuous action of a separable completely metrizable group can be reduced to the isomorphism of simple, separable AI $C^{*}$-algebras.
\end{abstract}

Mathematics Subject Classification 03E15 - 46L35 - 46A55

\section{Introduction}

Broadly speaking, a problem $P$ in a class $\Gamma$ is called complete in $\Gamma$ if any other problem in $\Gamma$ can be reduced to $P$. Complete problems typically appear

This research was partially supported by the NCN (the Polish National Science Centre) Grant no. 2012/05/D/ST1/03206, by the Foundation for Polish Science and by MNiSW (the Polish Ministry of Science and Higher Education) Grants no. 0435/IP3/2013/72 and N N201 418939.

$\bowtie$ Marcin Sabok

marcin.sabok@mcgill.ca

1 Department of Mathematics and Statistics, McGill University, 805 Sherbrooke Street West, Montreal H3A 0B9, Canada

2 Instytut Matematyczny Polskiej Akademii Nauk, ul. Śniadeckich 8, 00-956 Warszawa, Poland 
in logic and computer science, perhaps with the most prominent examples of NP-complete problems.

In the continuous setting, a descriptive set-theoretic complexity theory for problems arising as Borel and analytic equivalence relations on standard Borel spaces, has been developed by Kechris, Louveau, Hjorth and others [2,38,40, $41,48]$ over the last 30 years. The classification problems arising in this setting are of the following form: given an analytic or Borel equivalence relation $E$ on a standard Borel space $X$, decide whether two points in $X$ are $E$-equivalent. Of great interest here are the equivalence relations given by Borel actions of separable completely metrizable (i.e. Polish) groups on standard Borel spaces or, equivalently, continuous actions of Polish groups on Polish spaces (see [8]). Typically, isomorphism problems arising in various areas of mathematics are easily translated into this language. The relative complexity is measured in terms of Borel reducibility: an equivalence relation $E$ on $X$ is Borel reducible to an equivalence relation $F$ on $Y$ if there is a Borel map $f: X \rightarrow Y$ such that $x_{1} E x_{2}$ if and only if $f\left(x_{1}\right) F f\left(x_{2}\right)$ for every $x_{1}, x_{2} \in X$. The meaning of this notion is that the function $f$, being computable (Borel), gives a way of reducing the problem of $E$-equivalence of points in $X$ to that of $F$-equivalence of points in $Y$. We say that an equivalence relation $E$ is complete in a class $\Gamma$ of equivalence relations if it belongs to $\Gamma$ and every relation $F$ in $\Gamma$ is Borelreducible to $E$. For examples of complete analytic equivalence relations see [29,56]. Two relations $E$ and $F$ are bi-reducible if $E$ is Borel reducible to $F$ and $F$ is Borel reducible to $E$. For countable group actions, also a stronger notion (in the measure-theoretic context) is used: two group actions on standard Borel measure spaces $X$ and $Y$ are called orbit equivalent if there is a Borel isomorphism of $X$ and $Y$ which maps (a.e.) orbits to orbits (see [32]). We say that an equivalence relation is an orbit equivalence relation if it is bi-reducible with an equivalence relation induced by a Borel action of a Polish group. Descriptive set-theoretic complexity theory has enjoyed spectacular successes, for instance the striking results of Thomas $[66,67]$ on the relative complexity of isomorphism problems for torsion-free abelian groups or the results of Foreman, Rudolph and Weiss [31] on the conjugacy problem in ergodic theory.

The isomorphism problem for separable $\mathrm{C}^{*}$-algebras has been studied since the work of Glimm in the 1960's and evolved into the Elliott program that classifies $C^{*}$-algebras via their $K$-theoretic invariants. Glimm's result [35], restated in modern language, implies that the isomorphism relation for UHF algebras is smooth (see [33, Chapter 5.4]). In the 1970's the classification has been pushed forward to AF algebras via the $K_{0}$ group [17]. The Elliott invariant, which consists of the groups $K_{0}$ and $K_{1}$ together with the tracial simplex and the pairing map, was conjectured (see $[19,24]$ ) to completely classify all infinite-dimensional, separable, simple nuclear $\mathrm{C}^{*}$-algebras. The conjecture has been verified for various classes of $\mathrm{C}^{*}$-algebras, e.g certain classes of 
real rank zero algebras, $\mathrm{AH}$ algebras of slow dimension growth or separable, simple, purely infinite, nuclear algebras (modulo the universal coefficient theorem) $[18,21-23,49,50,64]$ and there have been dramatic breakthroughs in the program, including the counterexamples to the general classification conjecture constructed by Rørdam [65] and Toms [69].

The classification program of separable $C^{*}$-algebras can be studied from the point of view of descriptive set-theoretic complexity theory ( $\mathrm{cf}[20]$ ). The framework here has been set up in 1996 by Kechris [47] and more recently by Farah, Toms and Törnquist $[27,28]$.

The following question has become famous.

Question 1.1 (Farah et al, $[1,14,16,26,28]$ ) What is the complexity of the isomorphism relation for separable (nuclear, simple) $\mathrm{C}^{*}$-algebras?

The question is strictly connected with a long-standing conjecture (posed in the late 1990's) of Gao, Hjorth and Kechris.

Conjecture 1.2 (Hjorth [40], Gao-Kechris [34]) The homeomorphism relation of compact metric spaces is a complete orbit equivalence relation.

For some background on these problems the reader is advised to consult the lecture $^{1}$ of Farah at the ICM in Seoul [25].

In this paper we answer Question 1.1.

Theorem 1.3 The isomorphism relation of separable $C^{*}$-algebras is complete in the class of orbit equivalence relations. In fact, already the isomorphism of separable simple AI $C^{*}$-algebras is a complete orbit equivalence relation.

We would like to note here that very recently Zielinski [72] used the results of this paper (Theorem 1.4 below) to give a very elegant proof of Conjecture 1.2.

Question 1.1 appears on a 2008 problem list of Farah [26]. Since then, it has been a focus of intense research and several workshops have been devoted to this question (at AIM, BIRS, Luminy, Singapore, and others). Question 1.1 appears on a problem list supported by the American Institute of Mathematics [1, Problem 7.2] and on the list edited by Effros, Elliott, Farah and Toms [14, Problem (3)]. Also recently, it has been stated in several forms as [28, Problem 9.3], [28, Problem 9.7] (asked by Hjorth), [16, Question 4.1], [16, Question 4.2].

A lower bound on the complexity of the isomorphism of separable simple AI algebras has been obtained by Farah, Toms and Törnquist [28], who showed that the isomorphism of separable simple AI algebras is not classifiable by countable structures (see [33, Chapter 10]). The upper bound showing that it is an orbit equivalence relation has been proved recently by Elliott, Farah,

1 Which also includes a discussion of the results of the present paper. 
Paulsen, Rosendal, Toms, and Törnquist [16] using the technique of Gao and Kechris [34].

One has to mention here that an important point in the $K$-theoretic classification of separable simple nuclear $\mathrm{C}^{*}$-algebras is the functoriality of the invariants. While the theory of functorial reductions is still to be developed in the context of descriptive set theoretic complexity theory, it is worth noting that our proof of Theorem 1.3 shows features of functoriality. The details are discussed in Sect. 9.

Using the results of Thomsen [68], Farah, Toms and Törnquist [28] showed that the relation of affine homeomorphism of Choquet simplices is Borel reducible to the isomorphism relation of simple, separable, AI algebras. Theorem 1.3 will thus follow from the following.

Theorem 1.4 The relation of affine homeomorphism of Choquet simplices is complete in the class of orbit equivalence relations.

Gao and Kechris [34] and, independently, Clemens showed that the isometry relation of separable complete metric spaces is a complete orbit equivalence relation. Our proof of Theorem 1.4 reduces the relation of isometry of separable complete metric spaces to the affine homeomorphism of Choquet simplices.

The proof of Theorem 1.4 will be geometric. It will be based on two main new ideas connecting metrics on separable metric spaces with the affine geometry of Choquet simplices. In the first part of the proof we construct Choquet simplices using specific families of Lipschitz functions defined on a separable metric space. Here, we additionally incorporate an idea coming from the Katětov construction of the universal Urysohn space and use it in the analysis of the extreme boundary in our construction. In the second part, we introduce the blow-up construction that rigidifies the structure of a simplex with respect to a specific metric defined on its extreme points. This is later used to encode a metric into the affine structure of a simplex. The final construction is a fusion of these two methods.

It is worth noting here that Haydon [39] (see also [10, Theorem 29.9] and [13, Page 143]) showed that any Polish space is homeomorphic to the extreme bondary of a simplex. One should note, however, that this construction is not unique and, indeed, there are simplices with homeomorphic extreme boundaries which are not affinely homeomorphic (see [3, Page 119]). The general problem of determining a simplex from the structure of its extreme boundary is called the Dirichlet extension problem and is solved in [4]. In this paper, we take a different approach and, given a separable metric space, we construct a simplex whose extreme boundary only contains the metric space as a subspace. The advantage over Haydon's construction is that this construction is invariant under the isometry. Our simplices, which we call S-extensions of 
metric spaces, seem to be different from most of the typical constructions of simplices appearing in the literature.

This paper is organized as follows. In Sect. 2 we recall some basic facts from convex analysis and Choquet's theory. In Sect. 3 we recall and slightly strengthen the results of Clemens, Gao and Kechris on the isometry of separable metric spaces. Section 4 contains some elementary back-and-forth constructions for building affine homeomorphism. The $S$-extension construction appears in Sect. 5. Sections 6 and 7 form a preparation for a coding construction of metrics. The main construction appears in Sect. 8. Section 9 contains some concluding remarks and questions.

\section{Convex analysis and Choquet's theory}

For basic concepts of convex analysis and Choquet's theory we refer the reader to [44, Chapter 15] and to [5,6,10,62]. In this paper we use the terms simplex and Choquet simplex interchangeably and all convex compact sets that we consider are metrizable.

We consider the Hilbert cube $[0,1]^{\mathbb{N}}$ as a compact convex subset of a locally convex topological vector space (e.g. $\ell_{\infty}$ with the weak* topology) and with the standard metric on $[0,1]^{\mathbb{N}}$ given by $d_{[0,1]^{\mathbb{N}}}(x, y)=\sum_{n} 2^{-n}|x(n)-y(n)|$. Given a set $A$ in a locally convex vector topological space, we write $\operatorname{conv}(A)$ for the closed convex hull of $A$. For a compact convex set $C$, we write $\operatorname{ext}(C)$ for the set of extreme points of $C$. Given two convex compact sets $C$ and $D$, we write $C \simeq D$ to denote that $C$ and $D$ are affinely homeomorphic. We write $\Delta^{n}$ for the $n$-dimensional simplex. The Poulsen simplex [63] is the unique (see [54]) metrizable Choquet simplex with dense extreme boundary.

Whenever we consider a metric on a topological space, we assume it is compatible with the topology. Although convex compact sets are typically considered only with the affine and topological structure, we will sometimes use metrics on convex compact sets in locally convex vector topological spaces. We will always assume that if $C$ is a compact convex set with a metric $d_{C}$ on it, then there is a bigger convex compact set $D$ with $C-C \subseteq D$ and a norm $\|\cdot\|$ on $D$ such that $d_{C}(x, y)=\|x-y\|$ for every $x, y \in C$. For convex compact subsets of the Hilbert cube, we can use the metric $d_{[0,1]^{\mathbb{N}}}$ and for subsets of $\mathbb{R}$ the standard distance on $\mathbb{R}$. Note that the open balls in such metrics are convex.

Given an inverse system $\left(S_{n}, \pi_{n}\right)$ of simplices (with $\pi_{n}: S_{n+1} \rightarrow S_{n}$ an affine continuous surjection) and $x \in \lim S_{n}$, we write $x\left\lceil S_{i}\right.$ (or even $x\lceil i$ if it does not cause confusion) for the image of $x$ under the canonical projection map from $\lim S_{n}$ to $S_{i}$. Sometimes, we use the notation $x\left\lceil S_{i}\right.$ for $x \in S_{j}$ with $j>i$. It is worth noting here that, in general, it is not true that any continuous affine surjection is open and the exact characterization of when this happens has been given by Vesterstrøm [71]. The inverse limit of a system of simplices 
is again a simplex [43] and Lazar and Lindenstrauss [52] proved that any (metrizable) simplex is an inverse limit of a sequence of finite-dimensional simplices. Recently, López-Abad and Todorčević [55] showed that a generic inverse limit of simplices is affinely homeomorphic to the Poulsen simplex. For more on the Poulsen simplex we refer the reader to [44, Chapter 15] and [6, Chapter 3 Section 7].

The analysis on convex compact sets is dual to the theory of order unit Banach spaces via the spaces of affine functions (see [6, Chapter 2] and [15]).

The standard Borel structure on the space of simplices was introduced in [28] and is based on the parametrization of simplices proved by Lazar and Lindenstrauss [52] and the duality to the order unit spaces. Alternately, one can also use the induced Borel structure from the space of compact subsets of the Hilbert cube (see [28, Section 4.1.4]) or of the Poulsen simplex. Note that for a convex compact set $C$, the space of compact convex subsets of $C$ is closed in $K(C)$. The space of simplices contained in $C$ is not closed but it is Borel in $K(C)$ [28, Lemma 4.7].

We will need the following simple observation about affine continuous functions on convex compact sets. It is proved by an obvious averaging argument.

Proposition 2.1 Let $C, D$ be convex compact sets in locally convex vector topological spaces and let $d_{C}$ be a metric on $C$. If $f, g: D \rightarrow C$ are two affine continuous functions such that $d_{C}(f(e), g(e)) \leq \varepsilon$ for some $\varepsilon>0$ and everye $\in \operatorname{ext}(D)$, then $\|f-g\|_{\infty} \leq \varepsilon$.

\section{Isometry of perfect Polish spaces of bounded diameter}

Recall that the Urysohn space $\mathbb{U}[70]$ is the universal ultrahomogeneous separable metric space, and for every $r>0$ the bounded Urysohn space $\mathbb{U}_{1}$ is its counterpart of diameter 1 . Both these spaces are constructed in the same way and have finite isometry extension properties. Also, in the literature, $\mathbb{U}_{1}$ is referred to as the Urysohn sphere and, as noted by Melleray, it is isometric to the sphere of diameter 1 (around any point) in the Urysohn space. For more on the Urysohn space and sphere, see [61, Chapter 5] or [53,59,60].

The standard Borel space of separable complete metric spaces is identified with the space of closed subsets of the Urysohn space $\mathbb{U}$ with its Effros Borel structure. Gao and Kechris [34], and independently Clemens [11,12], proved that the isometry relation on the space of separable complete metric spaces is complete in the class of orbit equivalence relations.

Similarly, we consider the standard Borel space of separable complete metric spaces of diameter bounded by 1 as the space of closed subsets of the Urysohn sphere $\mathbb{U}_{1}$.

For a Polish space $X$, write $F_{p}(X)$ for the space of perfect (nonempty) subsets of $X$. Note that if $X$ is compact, then $F_{p}(X)$ is $G_{\delta}$ in $K(X)$, which 
implies that for every Polish space $X$ the set $F_{p}(X)$ is Borel in $F(X)$. Given this, the standard Borel space of perfect separable metric spaces of diameter bounded by 1 is identified with $F_{p}\left(\mathbb{U}_{1}\right)$.

The following result is essentially due to Gao, Kechris and Clemens, and can be proved by an easy modification of either the proof of Gao and Kechris [34] or of the proof of Clemens [11]. Below we present a nice and short proof suggested by the Referee.

Proposition 3.1 The isometry relation of perfect spaces of diameter less than 1 is complete in the class of orbit equivalence relations.

Proof Embed the Banach space $C\left(2^{\mathbb{N}}\right)$, of continuous functions on the Cantor set, isometrically (as a metric space) into the Urysohn space $\mathbb{U}$. This embedding induces a Borel map $\Lambda$ from the space of closed subspaces of $C\left(2^{\mathbb{N}}\right)$ to $F_{p}(\mathbb{U})$ (as every Banach space is perfect). The map $\Lambda$ is simply taking separable Banach spaces and viewing them as metric spaces. By the work of Melleray [58], the relation of linear isometry between separable Banach spaces is complete in the class of orbit equivalence relations. As every surjective isometry between Banach spaces is affine, the map $\Lambda$ is a reduction from linear isometry of separable Banach spaces to the isometry of perfect metric spaces. So the latter is also complete in the class of orbit equivalence relations.

Finally, consider the map $\left(X, d_{X}\right) \mapsto\left(X, \frac{d_{X}}{1+d_{X}}\right)$ and note that it is a reduction from the isometry relation of perfect metric spaces to the isometry relation of perfect metric spaces of diameter less than 1.

Gao and Kechris [34, Section 2D] show also that the isometry relation of subspaces of $\mathbb{U}$ is bireducible with the equivalence relation induced by the action of Iso(U) on $F(\mathbb{U})$. They deduce it from the fact that for every separable complete metric space $X$ there is an isometric copy $Z(X)$ of $X$ in $\mathbb{U}$ such that $F(\mathbb{U}) \ni X \mapsto Z(X) \in F(\mathbb{U})$ is Borel and for every $X, Y \in F(\mathbb{U})$ if $X$ and $Y$ are isometric, then there is an isometry $\varphi \in \operatorname{Iso}(\mathbb{U})$ such that $\varphi^{\prime \prime} Z(X)=Z(Y)$. This follows from the fact (see Gromov [36, Page 79] and [34, Lemma 2.2]) that for every separable metric space there is a metric extension $X^{*}$ of $X$ (obtained via the Katětov construction) that is canonically isometric to the Urysohn space $\mathbb{U}$ and such that any isometry $\varphi: X \rightarrow Y$ extends to an isometry $\varphi^{*}: X^{*} \rightarrow Y^{*}$. For more details on this construction see [33, Page 325]. Exactly the same arguments apply to metric spaces of diameter bounded by 1 and the Urysohn sphere $\mathbb{U}_{1}$, which gives the following.

Proposition 3.2 There is a Borel map $Z: F_{p}\left(\mathbb{U}_{1}\right) \rightarrow F_{p}\left(\mathbb{U}_{1}\right)$ such that for every $X \in F_{p}\left(\mathbb{U}_{1}\right)$ there exists an isometry $\theta_{X}: X \rightarrow Z(X)$ and if $X, Y \in F_{p}\left(\mathbb{U}_{1}\right)$ are isometric via an isometry $\varphi: X \rightarrow Y$, then there is an isometry $\tilde{\varphi} \in \operatorname{Iso}\left(\mathbb{U}_{1}\right)$ with $\tilde{\varphi}^{\prime \prime} Z(X)=Z(Y)$ such that $\tilde{\varphi}$ extends $\theta_{Y} \varphi\left(\theta_{X}\right)^{-1}$. 


\section{Approximate intertwinings}

Say that an inverse system $\left(S_{n}, \pi_{n}: n \in \mathbb{N}\right)$ of simplices is increasing if $S_{n} \subseteq S_{n+1}$ is a face of $S_{n+1}$ for each $n \in \mathbb{N}$ and $\pi_{n}(s)=s$ for each $s \in S_{n}$. Note that if $\left(S_{n}: n \in \mathbb{N}\right)$ is an increasing system of simplices, then also $S_{i} \subseteq \lim _{\longleftarrow} S_{n}$ is a face of $\lim _{\leftarrow} S_{n}$, for each $i \in \mathbb{N}$.

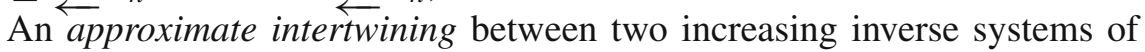
simplices $\left(S_{i}: i \in \mathbb{N}\right)$ and $\left(T_{i}: i \in \mathbb{N}\right)$ is a sequence of affine continuous injective maps $\varphi_{i}: S_{n_{i}} \rightarrow T_{m_{i}}$ and $\psi_{i}: T_{m_{i}} \rightarrow S_{n_{i+1}}$ (for some increasing sequences $n_{i}$ and $m_{i}$ of natural numbers) such that $\varphi_{n} \subseteq \psi_{n}{ }^{-1}, \psi_{n} \subseteq \varphi_{n+1}{ }^{-1}$ (recall that the systems are increasing) and for each $\varepsilon>0$ there exists $i$ such that for each $j>i$ and for each $x \in S_{n_{j}}$ and $y \in T_{m_{j}}$ we have

- $d_{T_{m_{i}}}\left(\varphi_{i}\left(x\left\lceil S_{n_{i}}\right), \varphi_{j}(x)\left\lceil T_{m_{i}}\right)<\varepsilon\right.\right.$,

- $d_{S_{n_{i+1}}}\left(\psi_{i}\left(y\left\lceil T_{m_{i}}\right), \psi_{j}(y)\left\lceil S_{n_{i+1}}\right)<\varepsilon\right.\right.$.

Proposition 4.1 Suppose $\left(S_{i}: i \in \mathbb{N}\right)$ and $\left(T_{i}: i \in \mathbb{N}\right)$ are two increasing inverse systems of simplices and there is an approximate intertwining between these systems. Then $\lim S_{i}$ and $\lim T_{i}$ are affinely homeomorphic via a map which extends all the maps in the approximate intertwining.

Proof This is a back-and-forth argument. Write $S=\lim S_{i}$ and $T=\lim T_{i}$. Let $\left(\varphi_{n}: n \in \mathbb{N}\right)$ and $\left(\psi_{n}: n \in \mathbb{N}\right)$ form an approximate intertwining and for simplicity assume that $n_{i}=m_{i}=i$. For $x \in S$ and $n<k$ look at the point $\varphi_{k}\left(x\left\lceil S_{k}\right)\left\lceil T_{n}\right.\right.$ which is obtained by first projecting $x$ to $S_{k}$, then applying $\varphi_{k}$ and finally projecting to $T_{n}$. Note that for each $x \in S$ and for each $n \in \mathbb{N}$ the sequence of points $\left(\varphi_{k}\left(x\left\lceil S_{k}\right)\left\lceil T_{n}: k \in \mathbb{N}\right)\right.\right.$ in $T_{n}$ is Cauchy, by the property of approximate intertwining. For $x \in S$ write $x_{n}=\lim _{k} \varphi_{k}\left(x\left\lceil S_{k}\right) \mid T_{n}\right.$ for the limit of this sequence. Note that for each $n \in \mathbb{N}$ the point $x_{n}$ belongs to $T_{n}$ and given $n<m \in \mathbb{N}$ we have $x_{m}\left\lceil T_{n}=x_{n}\right.$ since $\left(\lim _{k} \varphi_{k}\left(x\left\lceil S_{k}\right)\left\lceil T_{m}\right)\left\lceil T_{n}=\right.\right.\right.$ $\lim _{k}\left(\varphi_{k}\left(x\left\lceil S_{k}\right)\left\lceil T_{m}\right)\left\lceil T_{n}\right)=\lim _{k} \varphi_{k}\left(x\left\lceil S_{k}\right)\left\lceil T_{n}\right.\right.\right.\right.$. Thus, for each $x \in S$ there exists a point $y \in T$ whose projection to $T_{n}$ is $x_{n}$, for every $n$. Write $\varphi(x)=y$. Note that the map $\varphi$ is affine since the composition of $\varphi$ with the projection to $T_{n}$ is affine (as the limit of affine maps) for each $n \in \mathbb{N}$. Note also that for each $n \in \mathbb{N}$ the restriction of $\varphi$ to $S_{n}$ is equal to $\varphi_{n}$ since if $x \in S_{n}$, then $\varphi_{k}\left(x\left\lceil S_{k}\right)=\varphi_{k}(x)=\varphi_{n}(x)\right.$ for each $k>n$.

Claim 4.2 The map $\varphi$ is continuous.

Proof Fix $\varepsilon>0$. We need $\delta>0$ such that if $x_{1}, x_{2} \in S$ are such that $d_{S}\left(x_{1}, x_{2}\right)<\delta$, then $d_{T}\left(\varphi\left(x_{1}\right), \varphi\left(x_{2}\right)\right)<\varepsilon$. Fix $n \in \mathbb{N}$ big enough so that $T_{n}$ is $\varepsilon / 3$-dense in $T$ and $\varphi(x)$ is $\varepsilon / 3$-close to $\varphi_{n}\left(x\left\lceil S_{n}\right)\right.$. Let $\delta>0$ be such that if $d_{S_{n}}\left(x_{1}, x_{2}\right)<\delta$, then $d_{T_{n}}\left(\varphi_{n}\left(x_{1}\right), \varphi_{n}\left(x_{2}\right)\right)<\varepsilon / 3$. Then, clearly, $\delta$ is as needed. 
Analogously define the map $\psi: T \rightarrow S$ and argue that it is affine and continuous. Now, the facts that $\bigcup_{n} S_{n}$ is dense in $S$ and $\bigcup_{n} T_{n}$ is dense in $T, \varphi$ extends $\varphi_{n}$ and $\psi$ extends $\psi_{n}$ for each $n \in \mathbb{N}$, imply that $\varphi^{-1}=\psi$, as $\varphi_{n} \subseteq \psi_{n}{ }^{-1}$ and $\psi_{n} \subseteq \varphi_{n+1}{ }^{-1}$.

\section{$5 S$-extensions of metric spaces}

In this section we define the $S$-extensions of metric spaces. The definitions below stem from a simple observation that any finite metric space $\left(X, d_{X}\right)$ is isometric to the extreme boundary of a convex compact subset of $\ell_{\infty}^{n}$ for some $n \in \mathbb{N}$. Indeed, if $X=\left\{x_{1}, \ldots, x_{n}\right\}$, then the map $x_{i} \mapsto a_{i}=$ $\left(d_{X}\left(x_{i}, x_{1}\right), \ldots, d_{X}\left(x_{i}, x_{n}\right)\right)$ maps $X$ isometrically into the extreme boundary of the convex hull of $\left\{a_{i}: i \leq n\right\}$ taken with the $\ell_{\infty}$ metric.

Given a sequence $C_{n}$ of compact convex subsets of a compact convex set $C$ in a locally convex topological vector space, say that $C_{n}$ is convergent if it convergent in $K(C)$ (i.e. in the Hausdorff metric) and write $\lim _{n} C_{n}$ for the limit. Note that any increasing sequence of compact convex sets is convergent and then $\lim _{n} C_{n}$ is equal to the closure of its union.

Given a metric space $\left(X, d_{X}\right)$, together with its (enumerated) countable dense set $D \subseteq X$ and a countable (enumerated) family $F \subseteq C(X,[0,1])$ of continuous functions with values in the unit interval, we will form a convex compact set $S\left(X, d_{X}, D, F\right)$. Let $D=\left(d_{n}: n \in \mathbb{N}\right)$ and $F=\left(f_{n}\right.$ : $n \in \mathbb{N})$. Consider the vectors $a_{n}=\left(f_{1}\left(d_{n}\right), f_{2}\left(d_{n}\right), \ldots\right) \in[0,1]^{\mathbb{N}}$ and let $S_{n}\left(X, d_{X}, D, F\right)$ be the convex hull of the set $\left\{a_{i}: i \leq n\right\}$ in $[0,1]^{\mathbb{N}}$ Note that the sequence $S_{n}\left(X, d_{X}, D, F\right)$ is increasing, hence convergent in $K\left([0,1]^{\mathbb{N}}\right)$.

Definition 5.1 Given a metric space $\left(X, d_{X}\right)$ define $S\left(X, d_{X}, D, F\right)$ as $\lim _{n \rightarrow \infty} S_{n}\left(X, d_{X}, D, F\right)$.

We will always consider $S\left(X, d_{X}, D, F\right)$ with the metric $d_{[0,1]^{\mathbb{N}}}$ induced from the Hilbert cube. Note that since all $S_{n}\left(X, d_{X}, D, F\right)$ 's are convex, so is $S\left(X, d_{X}, D, F\right)$. In general, this is all we know about this set. We will show, however, that if the functions $F$ are carefully chosen, then $S\left(X, d_{X}, D, F\right)$ is a simplex and its set of extreme points contains a dense homeomorphic copy of $X$.

For each $n$ write $S_{n}^{n}\left(X, d_{X}, D, F\right)$ for the projection of $S_{n}\left(X, d_{X}, D, F\right)$ into the first $n$-many coordinates. Treating $[0,1]^{n}$ as a subset of the Hilbert cube (by padding sequences with zeros) note that the Hilbert cube is the limit of the sets $[0,1]^{n}$. In the same way, $S_{n}^{n}\left(X, d_{X}, D, F\right)$ is treated as a subset of the Hilbert cube (also by padding sequences with zeros) and, under this identification, $S\left(X, d_{X}, D, F\right)$ is the limit of the sets $S_{n}^{n}\left(X, d_{X}, D, F\right)$.

We first show that the definition of $S\left(X, d_{X}, D, F\right)$ does not depend on the choice of the dense set $D$, in particular it does not depend on the enumeration 
of $D$. From the point of view of further applications, we would need the fact that if $D$ and $E$ are two countable dense sets in $X$, then $S\left(X, d_{X}, D, F\right)$ and $S\left(X, d_{X}, E, F\right)$ are affinely homeomorphic. However, they are actually the same set.

Lemma 5.2 If $F$ is a countable family of Lipschitz 1 functions and $D, E$ are two countable dense subsets of $X$, then $S\left(X, d_{X}, D, F\right)$ and $S\left(X, d_{X}, E, F\right)$ are equal.

Proof This is straightforward. Using the fact that both $D$ and $E$ are dense in $X$, conclude that the Hausdorff distance of $S\left(X, d_{X}, D, F\right)$ and $S\left(X, d_{X}, E, F\right)$ is arbitrarily small. Since $S\left(X, d_{X}, D, F\right)$ and $S\left(X, d_{X}, E, F\right)$ are compact, they must be then equal.

In the sequel, we write $S\left(X, d_{X}, F\right)$ rather than $S\left(X, d_{X}, D, F\right)$. Another way of stating the previous lemma is then to say that $S\left(X, d_{X}, F\right)$ is the closed convex hull of the set $\left\{\left(f_{1}(x), f_{2}(x), \ldots\right): x \in X\right\}$. In principle, this can be taken as the definition of $S\left(X, d_{X}, F\right)$ but in further arguments we will use the approximation of $S\left(X, d_{X}, F\right)$ by $S_{n}\left(X, d_{X}, D, F\right)$.

Definition 5.3 We say that a countable family $F$ of Lipschitz 1 functions on a metric space $\left(X, d_{X}\right)$ is saturated if for every $x \in X$ the distance function $z \mapsto d_{X}(z, x)$ belongs to the uniform closure of $F$.

Note that if $D$ is a dense subset of $X$, then the family of distance functions to the points from $D$ is always saturated. Similarly, if $X$ is embedded into the Urysohn space and $D$ is a fixed dense subset of the Urysohn space, then the family of distance functions (defined on $X$ ) to the points in $D$ is also saturated. In the main part of the proof we will use saturated families of this form, however, some facts below will be stated for arbitrary saturated families, in sake of generality.

Note that $D$ can be seen as a subset of $S\left(X, d_{X}, D, F\right)$ via the map $d \mapsto$ $\left(f_{1}(d), f_{2}(d), \ldots\right)$. Note also that since all distance functions to the points of $D$ are in the uniform closure of $F$, the above map is an embedding. Denote this map by $i_{F}^{D}$.

Lemma 5.4 If $F$ is saturated, then there is a canonical homeomorphic embedding $i_{F}$ of $X$ into $S\left(X, d_{X}, F\right)$ which extends $i_{F}^{D}$ for all countable dense $D \subseteq X$ and is Lipschitz 1.

Proof Write $i_{F}(x)=\left(f_{1}(x), f_{2}(x), \ldots\right)$ and note that $i_{F}(x) \in S\left(X, d_{X}, F\right)$ for every $x \in X$ by Lemma 5.2. It is clear that, when viewed as a map to $S\left(X, d_{X}, D, F\right), i_{F}$ extends $i_{F}^{D}$. The fact that $i_{F}$ is an embedding of $X$ into $S\left(X, d_{X}, D, F\right)$ follows from the fact that all distance functions to the points in $D$ are in the uniform closure of $F$. 
To see that $i_{F}$ is a homeomorphism, suppose that $x_{n} \in X$ and $x \in X$ are such that $i_{F}\left(x_{n}\right) \rightarrow i_{F}(x)$. This means that $f\left(x_{n}\right) \rightarrow f(x)$ for every $f \in F$. Again, since the distance functions from all points in $X$ are in the uniform closure of $F$ we have $d\left(x_{n}, y\right) \rightarrow d(x, y)$ for every $y \in X$ and hence $x_{n} \rightarrow x$, which shows that $i_{F}$ is a homeomorphism.

Finally, the fact that $i_{F}$ is Lipschitz 1 follows immediately from the fact that all functions in $F$ are Lipschitz 1.

In the sequel we will abuse notation and treat $X$ as a subset of $S\left(X, d_{X}, F\right)$, unless this can cause confusion.

Now, we need to locate the extreme points of the sets $S\left(X, d_{X}, F\right)$. Notice that if $F_{1} \subseteq F_{2}$ are two families of Lipschitz 1 functions, then there is a natural affine continuous projection from $S\left(X, d_{X}, F_{2}\right)$ to $S\left(X, d_{X}, F_{1}\right)$ (which forgets the coordinates from $F_{2} \backslash F_{1}$ ).

Note also that if $K$ and $L$ are convex compact sets and $\varphi: K \rightarrow L$ is an affine continuous surjection, then $\operatorname{ext}(L) \subseteq \varphi^{\prime \prime} \operatorname{ext}(K)$. This implies that the larger the family $F$ we take, the more extreme points we get in $S\left(X, d_{X}, F\right)$.

Lemma 5.5 If $F$ is saturated, then the points of $X$ are extreme points in $S\left(X, d_{X}, F\right)$.

Given a compact convex set $C$ in a locally convex vector topological space, with a metric $d_{C}$ on $C$, and a subset $A \subseteq C$ we say that $A$ is an $\varepsilon$-face provided that for every $x, y \in C$ if $\frac{1}{2}(x+y) \in A$, then both $d_{C}(x, A)$ and $d_{C}(y, A)$ are smaller than $\varepsilon$. Note that if $A \subseteq C$ is written as $A=\bigcap_{n} A_{n}$ so that each $A_{n}$ is closed convex and for each $\varepsilon>0$ there is $n_{0}$ such that $A_{n}$ is an $\varepsilon$-face for each $n>n_{0}$, then $A$ is a face.

Proof of Lemma 5.5 We will again look at $S\left(X, d_{X}, D, F\right)$ for a fixed countable dense set $D \subseteq X$. By Proposition 5.2, it is enough to show that the points of $D$ are extreme in $S\left(X, d_{X}, D, F\right)$. Moreover, it is enough to show that given the enumeration of $D$ as $\left(d_{1}, d_{2}, \ldots\right)$, the point $d_{1}$ is extreme in $S\left(X, d_{X}, D, F\right)$.

Claim 5.6 For any $A \subseteq X$ we have $\operatorname{diam}_{S\left(X, d_{X}, F\right)}\left(\operatorname{conv}\left(i_{F} A\right)\right) \leq \operatorname{diam}_{X}(A)$.

Proof This follows directly from the fact that $i_{F}$ is Lipschitz 1 and from local convexity of the Hilbert cube.

Write $A_{n}$ for the closed convex hull of the ball in $X$ around $d_{1}$ of diameter $1 / n$. Claim 5.6 implies that $\bigcap_{n} A_{n}$ contains only one point, and thus is the singleton $\left\{d_{1}\right\}$. We will show that for each $\varepsilon>0$ there is $n_{0}$ such that $A_{n}$ is an $\varepsilon$-face for every $n>n_{0}$.

Fix $\varepsilon>0$ and find a function $f \in F$ which is $\varepsilon / 8$-uniformly close to the distance function $z \mapsto d\left(z, d_{1}\right)$. Without loss of generality assume that $f=f_{1}$. 
Suppose now that $n>8 / \varepsilon$ and $y, z \in S\left(X, d_{X}, F\right)$ are such that $\frac{1}{2}(y+z) \in$ $A_{n}$. Approximate $y$ with $y^{\prime}$ and $z$ with $z^{\prime}$ such that $d_{S\left(X, d_{X}, F\right)}\left(y, y^{\prime}\right)<\varepsilon / 8$, $d_{S\left(X, d_{X}, F\right)}\left(z, z^{\prime}\right)<\varepsilon / 8$ and $y^{\prime}, z^{\prime} \in S_{k}\left(X, d_{X}, D, F\right)$ for some $k \in \mathbb{N}$. Note that then $\frac{1}{2}\left(y^{\prime}+z^{\prime}\right)$ is $\varepsilon / 8$-close to $A_{n}$. Since the first coordinate of every point in $A_{n}$ is smaller than $1 / n+\varepsilon / 8$, the first coordinate of $\frac{1}{2}\left(y^{\prime}+z^{\prime}\right)$ is smaller than $1 / n+\varepsilon / 4$. Hence, the first coordinates of both $y^{\prime}$ and $z^{\prime}$ are smaller than $2(1 / n+\varepsilon / 4)$, which is smaller than $3 \varepsilon / 4$. Recall that we have

$$
\left\|f_{1}-d_{X}\left(\cdot, d_{1}\right)\right\|_{\infty}<\varepsilon / 8
$$

Suppose that

$$
y^{\prime}=\left(\sum_{i=1}^{k} \alpha_{i} f_{1}\left(d_{i}\right), \sum_{i=1}^{k} \alpha_{i} f_{2}\left(d_{i}\right), \ldots\right)
$$

for some $\alpha_{1}, \ldots, \alpha_{k} \geq 0$ with $\sum_{i=1}^{k} \alpha_{i}=1$. Since $\sum_{i=1}^{k} \alpha_{i} f_{1}\left(d_{i}\right) \leq \frac{3 \varepsilon}{4}$, by (1) we have $\sum_{i=1}^{k} \alpha_{i} d_{X}\left(d_{i}, d_{1}\right) \leq \frac{3 \varepsilon}{4}+\frac{\varepsilon}{8}$. Now, for each $j$ the function $f_{j}$ is Lipschitz 1, so

$$
\left|f_{j}\left(d_{i}\right)-f_{j}\left(d_{1}\right)\right| \leq d_{X}\left(d_{i}, d_{1}\right) .
$$

Recall that the point $d_{1}$ is identified with the sequence $\left(f_{1}\left(d_{1}\right), f_{2}\left(d_{1}\right), \ldots\right)$. With this in mind, by (2) for each $j$ we have

$$
\begin{aligned}
\left|y^{\prime}(j)-d_{1}(j)\right| & =\left|\sum_{i=1}^{k} \alpha_{i} f_{j}\left(d_{i}\right)-f_{j}\left(d_{1}\right)\right| \\
& =\left|\sum_{i=1}^{k} \alpha_{i} f_{j}\left(d_{i}\right)-\sum_{i=1}^{k} \alpha_{i} f_{j}\left(d_{1}\right)\right| \\
& \leq \sum_{i=1}^{k} \alpha_{i} d_{X}\left(d_{i}, d_{1}\right) \leq \frac{3 \varepsilon}{4}+\frac{\varepsilon}{8}
\end{aligned}
$$

This, and an analogous argument for $z^{\prime}$ in place of $y^{\prime}$, shows that

$$
d_{S\left(X, d_{X}, F\right)}\left(y^{\prime}, d_{1}\right)<\frac{3 \varepsilon}{4}+\frac{\varepsilon}{8} \quad \text { as well as } \quad d_{S\left(X, d_{X}, F\right)}\left(z^{\prime}, d_{1}\right)<\frac{3 \varepsilon}{4}+\frac{\varepsilon}{8} .
$$

This implies that $d_{S\left(X, d_{X}, F\right)}\left(y, d_{1}\right)<\frac{3 \varepsilon}{4}+\frac{\varepsilon}{4}=\varepsilon$ and $d_{S\left(X, d_{X}, F\right)}\left(z, d_{1}\right)<$ $\frac{3 \varepsilon}{4}+\frac{\varepsilon}{4}=\varepsilon$, which shows that $A_{n}$ is an $\varepsilon$-face.

The fact that $X$ is dense in the set of extreme points of $S\left(X, d_{X}, F\right)$ will follow from the following general lemma. 
Lemma 5.7 Suppose $C_{n}$ is an increasing sequence of compact convex subsets of a metrizable convex compact set $C$ in a locally convex topological vector space. Then $\operatorname{ext}\left(\lim _{n} C_{n}\right)$ is contained in the closure of $\bigcup_{n} \operatorname{ext}\left(C_{n}\right)$.

Proof Write $K$ for the closure of $\bigcup_{n} \operatorname{ext}\left(C_{n}\right)$ and suppose $x \in \lim _{n} C_{n}$ is such that $x \notin K$. We need to show that $x$ is not an extreme point of $\lim _{n} C_{n}$. Note that [44, Chapter 15, Proposition 2.3] $x$ is a barycenter of a probability measure $\mu$ concentrated on $K$. By a theorem of Bauer [7] (see also [62, Proposition 1.4]) if $x$ is an extreme point and a barycenter of a probability measure $\mu$, then $\mu=\delta_{x}$. But $\mu$ is concentrated on $K$, so cannot be equal to $\delta_{x}$.

This immediately gives the following.

Corollary 5.8 If $F$ is saturated, then $X$ is dense in the set of extreme points of $S\left(X, d_{X}, F\right)$.

Let us now see some examples of convex compact sets that can arise as $S\left(X, d_{X}, F\right)$. The next proposition will not be used later in the proof but it shows some ideas behind the constructions that follow.

In the following proposition, for a compact metric space $X$, write $P(X)$ for the Bauer simplex of all Borel probability measures on $X$. The extreme boundary of $P(X)$ is canonically homeomorphic to $X$ and any simplex whose extreme boundary is homeomorphic to $X$ is canonically affinely homeomorphic to $P(X)$ (this follows for example from the positive solution to the Dirichlet extension problem for Bauer simplices [4]).

As a comment to the assumption of the following proposition, note that if $\left(X, d_{X}\right)$ is compact, then Lipschitz functions are dense in $C(X)$, by the StoneWeierstrass theorem. Thus, if $\left(X, d_{X}\right)$ is compact, then the set of all Lipschitz 1 functions is linearly dense in $C(X)$.

Proposition 5.9 Suppose $\left(X, d_{X}\right)$ is compact, $F$ is saturated and linearly dense in $C(X)$. Then $S\left(X, d_{X}, F\right)$ is affinely homeomorphic to the Bauer simplex $P(X)$.

Proof Note that the set of extreme points of $S\left(X, d_{X}, F\right)$ is equal to $X$ by Lemma 5.5 and Corollary 5.8. We need to prove that $S\left(X, d_{X}, F\right)$ is a simplex. For that, suppose $\mu, v$ are two distinct probability measures on $X$. By linear density of $F$ in $C(X)$, there is $f \in F$ such that $\int f d \mu \neq \int f d \nu$. Without loss of generality, assume that $f=f_{1}$. Pick a countable dense set $D \subseteq X$ and choose two sequences of atomic measures $\mu_{n}$ and $v_{n}$ concentrated on $D$ such that $\mu_{n} \rightarrow \mu$ and $v_{n} \rightarrow v$. Note that each $\mu_{n}$ and $v_{n}$ has a barycenter in one of the sets $S_{k}\left(X, d_{X}, D, F\right)$, for if 


$$
\begin{gathered}
\mu_{n}=\sum_{i=1}^{k_{n}} \alpha_{i}^{n} \delta_{d_{i}^{n}} \text { with } \quad d_{i}^{n} \in D, \alpha_{i}^{n} \geq 0, \sum_{i=1}^{k_{n}} \alpha_{i}^{n}=1, \\
v_{n}=\sum_{i=1}^{l_{n}} \beta_{i}^{n} \delta_{e_{i}^{n}} \text { with } \quad e_{i}^{n} \in D, \beta_{i}^{n} \geq 0, \sum_{i=1}^{k_{n}} \beta_{i}^{n}=1,
\end{gathered}
$$

then the barycenter of $\mu_{n}$ is $\sum_{i=1}^{k_{n}} \alpha_{i}^{n} d_{i}^{n}$ and the barycenter of $v_{n}$ is $\sum_{i=1}^{l_{n}} \beta_{i}^{n} e_{i}^{n}$. Note that then $\sum_{i=1}^{k_{n}} \alpha_{i}^{n} f_{1}\left(d_{i}^{n}\right)$ and $\sum_{i=1}^{l_{n}} \beta_{i}^{n} f_{1}\left(e_{i}^{n}\right)$ are the first coordinates in $[0,1]^{\mathbb{N}}$ of the barycenters of $\mu_{n}$ and $v_{n}$. Thus, the first coordinate of the barycenter of $\mu$ is the limit of the first coordinates of the barycenters of $\mu_{n}$ [44, Chapter 15, Proposition 2.2] and is equal to

$$
\lim _{n} \sum_{i=1}^{k_{n}} \alpha_{i}^{n} f_{1}\left(d_{i}^{n}\right)=\lim _{n} \int f_{1} d \mu_{n}=\int f_{1} d \mu
$$

and the first coordinate of the barycenter of $v$ is the limit of the first coordinates of the barycenters of $v_{n}$ and is equal to

$$
\lim _{n} \sum_{i=1}^{l_{n}} \beta_{i}^{n} f_{1}\left(e_{i}^{n}\right)=\lim _{n} \int f_{1} d v_{n}=\int f_{1} d \nu .
$$

Thus, $\mu$ and $v$ have different barycenters, which shows that $S\left(X, d_{X}, F\right)$ is a simplex.

Now we need to see how $S\left(X, d_{X}, F\right)$ depends on the choice of $F$. First note that it does not depend on the enumeration of $F$.

Lemma 5.10 Suppose $F$ and $G$ enumerates the same set of functions as $F$. Then $S\left(X, d_{X}, F\right)$ and $S\left(X, d_{X}, G\right)$ are affinely homeomorphic.

Proof Let $\pi: \mathbb{N} \rightarrow \mathbb{N}$ be a permutation such that $G=\left(f_{\pi(1)}, f_{\pi(2)}, \ldots\right)$. Let $h:[0,1]^{\mathbb{N}} \rightarrow[0,1]^{\mathbb{N}}$ be defined as $h\left(x_{1}, x_{2}, \ldots\right)=\left(x_{\pi(1)}, x_{\pi(2)}, \ldots\right)$ and note that $h$ is an affine homeomorphism of the Hilbert cube which maps $S\left(X, d_{X}, F\right)$ to $S\left(X, d_{X}, G\right)$.

Suppose now that $\left(X, d_{X}\right)$ is a metric space, $D \subseteq X$ is a countable dense set and that $F$ consists of distance functions from the points in $D$. In such a case, we denote $F$ by $d_{X} D$. Note that $d_{X} D$ always consists of Lipschitz 1 functions and its uniform closure contains all distance functions to the points in $X$.

Proposition 5.11 Suppose $\left(X, d_{X}\right)$ is separable, complete and $D, E \subseteq X$ are two countable dense sets. Then there is an affine homeomorphism $\tau_{D}^{E}$ : 
$S\left(X, d_{X}, d_{X} D\right) \rightarrow S\left(X, d_{X}, d_{X} E\right)$ such that the following diagram commutes.

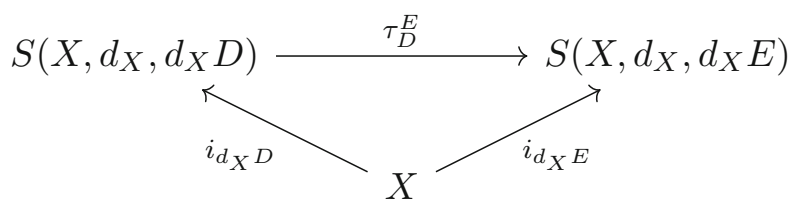

The map $\tau_{D}^{E}$ should be treated as the transition map between the coordinate systems of $D$ and $E$.

Proof First note that isolated points of $X$ must belong to both $D$ and $E$ and hence if $X_{1}$ is the set of isolated points, then by Lemma 5.10 we can assume that $X_{1}$ is enumerated in the same way in $D$ and $E$. Thus, without loss of generality we can assume that $X$ has no isolated points. Moreover, we can assume that $D$ and $E$ are disjoint since we can always use a third countable dense set which is disjoint from both $D$ and $E$. Write $D=\left(d_{0}, d_{1}, \ldots\right)$ and let $D^{\prime}=\left(d_{1}, d_{2}, \ldots\right)$.

Claim 5.12 $S\left(X, d_{X}, d_{X} D\right)$ and $S\left(X, d_{X}, d_{X} D^{\prime}\right)$ are affinely homeomorphic via a map $\tau_{D}^{D^{\prime}}: S\left(X, d_{X}, d_{X} D\right) \rightarrow S\left(X, d_{X}, d_{X} D^{\prime}\right)$ such that $\tau_{D}^{D^{\prime}} \circ i_{d_{X} D}=$ $i_{d_{X} D^{\prime}}$

Proof Write $C_{n}=S_{n}\left(X, d_{X}, D, d_{X} D\right)$ and $B_{n}=S_{n}\left(X, d_{X}, D, d_{X} D^{\prime}\right)$ for each $n \in \mathbb{N}$. Write also $[0,1]^{\mathbb{N}}$ as $[0,1]^{\{2,3, \ldots\}} \times[0,1]$ interpreting the second coordinate of the product as the first coordinate in the Hilbert cube. Pick a subsequence $d_{k_{n}} \rightarrow d_{0}$ in $D$ and note that the distance function to $d_{0}$ is the uniform limit of the distance functions to $d_{k_{n}}$ 's. By Proposition 2.1, this implies that on $\bigcup_{n} C_{n}$ the first coordinate is the uniform limit of the coordinates numbered with $k_{n}$ 's. Thus, $\bigcup_{n} C_{n}$ treated as a subset of $[0,1]^{\{2,3, \ldots\}} \times[0,1]$, is a graph of an affine function, say $a$, defined on $\bigcup_{n} B_{n}$. Note that since $a$ is the uniform limit of the sequence of functions given by coordinates in $\left\{k_{1}, k_{2}, \ldots\right\}$, it follows that this sequence of functions is uniformly Cauchy on $\bigcup_{n} B_{n}$. This sequence is also uniformly Cauchy on the closure of $\bigcup_{n} B_{n}$, and hence uniformly convergent. Thus, the function $a$ uniquely extends to an affine function defined on $\overline{\bigcup_{n} B_{n}}=S\left(X, d_{X}, d_{X} D^{\prime}\right)$. Note that the graph of this unique extension is equal to the closure of $\bigcup_{n} C_{n}$, i.e. $S\left(X, d_{X}, d_{X} D\right)$. Given that, the projection function from $[0,1]^{\{2,3, \ldots\}} \times[0,1]$ to $[0,1]^{\{2,3, \ldots\}}$ is an affine isomorphism from $S\left(X, d_{X}, d_{X} D\right)$ to $S\left(X, d_{X}, d_{X} D^{\prime}\right)$. Write $\tau_{D}^{D^{\prime}}$ for this isomorphism and note that since it just erases the first coordinate, we have $\tau_{D}^{D^{\prime}} \circ i_{d_{X} D}=i_{d_{X} D^{\prime}}$.

Write now $D_{n}$ for the sequence $\left(e_{1}, e_{2}, \ldots, e_{n}, d_{n+1}, d_{n+1}, \ldots\right)$.

Claim 5.13 For each $n \in \mathbb{N}$, there is an affine homeomorphism $\tau_{n}$ : $S\left(X, d_{X}, d_{X} D\right) \rightarrow S\left(X, d_{X}, d_{X} D_{n}\right)$ such that $\tau_{n} \circ i_{d_{X} D}=i_{d_{X} D_{n}}$. 
Proof It is enough to show that there is an affine homeomorphism $\tau_{n}^{\prime}$ : $S\left(X, d_{X}, d_{X} D_{n}\right) \rightarrow S\left(X, d_{X}, d_{X} D_{n+1}\right)$ with $\tau_{n}^{\prime} \circ i_{D_{n}}=i_{D_{n+1}}$ and without loss of generality assume that $n=0$. But this follows from Claim 5.12 since both $S\left(X, d_{X}, d_{X} D\right)$ and $S\left(X, d_{X}, d_{X} D_{1}\right)$ are affinely homeomorphic to $S\left(X, d_{X}, d_{X} D^{\prime}\right)$ via maps which make the appropriate diagrams commute.

Now note that since $\tau_{n+1} \tau_{n}^{-1}$ changes only the $n$th coordinate, the sequence $\tau_{n}$ is uniformly Cauchy with respect to the Hilbert cube metric, and thus converges to an affine map $\tau_{D}^{E}: S\left(X, d_{X}, d_{X} D\right) \rightarrow[0,1]^{\mathbb{N}}$. Moreover, since the distance of $\operatorname{rng} \tau_{n}$ to $S_{n}\left(X, d_{X}, D, d_{X} E\right)$ is smaller than $2^{-n}$, we have that $\tau_{D}^{E}$ is an affine map from $S\left(X, d_{X}, d_{X} D\right)$ to $S\left(X, d_{X}, d_{X} E\right)$. In the same way construct maps $\tau^{n}: S\left(X, d_{X}, d_{X} E\right) \rightarrow S\left(X, d_{X}, d_{X} E_{n}\right)$ with $E_{n}=$ $\left(d_{1}, d_{2}, \ldots, d_{n}, e_{n+1}, e_{n+2}, \ldots\right)$ and note that their limit is an affine function $\tau_{E}^{D}: S\left(X, d_{X}, d_{X} E\right) \rightarrow S\left(X, d_{X}, d_{X} D\right)$. The maps are clearly inverse to each other, so $\tau_{D}^{E}$ is an affine homeomorphism. Finally, $\tau_{D}^{E} \circ i_{d_{X} D}=i_{d_{X} E}$ follows from the fact that $\tau_{n} \circ i_{d_{X} D}=i_{d_{X} D_{n}}$ holds for each $n$ and $i_{d_{X} D_{n}} \rightarrow i_{d_{X} E}$. This ends the proof.

The following definition and proposition will not be used in the proof but we state it here since they may be useful in answering some questions posed in Sect. 9. Note that if we want the set $S\left(X, d_{X}, F\right)$ to be a simplex, a natural condition for the family $F$ is the following.

Definition 5.14 A countable family $F$ of Lipschitz 1 functions is called independent if for every $x_{1}, \ldots, x_{n} \in X$ there are $f_{1}, \ldots, f_{n} \in F$ such that the vectors $\left(f_{1}\left(x_{1}\right), \ldots, f_{n}\left(x_{1}\right)\right), \ldots,\left(f_{1}\left(x_{n}\right), \ldots, f_{n}\left(x_{n}\right)\right) \in \mathbb{R}^{n}$ are linearly independent.

The following proposition is stated for the Urysohn sphere $\mathbb{U}_{1}$ but it also holds true (with the same proof) for the Urysohn space $\mathbb{U}$. We say that a function $f: X \rightarrow[0, \infty)$ defined on a metric space $\left(X, d_{X}\right)$ is a Katětov function if for every $x, y \in X$ we have $|f(x)-f(y)| \leq d_{X}(x, y) \leq f(x)+f(y)$ (cf. [61, Lemma 5.1.22] and [33, Definition 1.2.1]).

Proposition 5.15 For any countable dense subset D of the Urysohn sphere $\mathbb{U}_{1}$, the set $d_{\mathbb{U}_{1}} D$ is independent.

Proof Write $d$ for the metric $d_{\mathbb{U}_{1}}$ on $\mathbb{U}_{1}$. Pick a countable dense set $D \subseteq \mathbb{U}_{1}$ and let $x_{1}, \ldots, x_{n} \in \mathbb{U}_{1}$. We show that there are $d_{1}, \ldots, d_{n} \in D$ such that the matrix

$$
\left(\begin{array}{ccc}
d\left(x_{1}, d_{1}\right) & \ldots & d\left(x_{1}, d_{n}\right) \\
\vdots & \ddots & \vdots \\
d\left(x_{n}, d_{1}\right) & \ldots & d\left(x_{n}, d_{n}\right)
\end{array}\right)
$$


is invertible. The proof is by induction. For $n=1$, any $d_{1} \neq x_{1}$ will do. Suppose $n>1$ and $x_{1}, \ldots, x_{n} \in \mathbb{U}$ are given. Pick any $d_{1}, \ldots, d_{n-1}$ that witness the inductive assumption for $x_{1}, \ldots, x_{n-1}$ and let $d^{\prime} \in D$ be such that $d\left(x_{n}, d^{\prime}\right)<d\left(x_{i}, d^{\prime}\right)$ for all $i \neq n$.. Consider the function

$$
\varepsilon \mapsto \operatorname{det}\left(\begin{array}{cccc}
d\left(x_{1}, d_{1}\right) & \ldots & d\left(x_{1}, d_{n-1}\right) & d\left(x_{1}, d^{\prime}\right) \\
\vdots & \ddots & \vdots & \vdots \\
d\left(x_{n}, d_{1}\right) & \ldots & d\left(x_{n}, d_{n-1}\right) & d\left(x_{n}, d^{\prime}\right)+\varepsilon
\end{array}\right)
$$

and note that it is a nonzero linear function, so there are arbitrarily small $\varepsilon>0$ at which it does not vanish. Pick such $\varepsilon_{0}>0$ which is smaller than $\min \left\{d\left(x_{i}, d^{\prime}\right)-d\left(x_{n}, d^{\prime}\right): i<n\right\}$. Note now that the function $f:\left\{x_{1}, \ldots, x_{n}\right\} \rightarrow[0,1]$ given by $f\left(x_{i}\right)=d\left(x_{i}, d^{\prime}\right)$ if $i<n$ and $f\left(x_{n}\right)=d\left(x_{n}, d^{\prime}\right)+\varepsilon_{0}$ is a Katětov function with values in $[0,1]$. Thus, there is $y \in \mathbb{U}_{1}$ which realizes $f$. This means that

$$
\operatorname{det}\left(\begin{array}{cccc}
d\left(x_{1}, d_{1}\right) & \ldots & d\left(x_{1}, d_{n-1}\right) & d\left(x_{1}, y\right) \\
\vdots & \ddots & \vdots & \vdots \\
d\left(x_{n}, d_{1}\right) & \ldots & d\left(x_{n}, d_{n-1}\right) & d\left(x_{n}, y\right)
\end{array}\right) \neq 0
$$

Since the set of such $y$ 's is clearly open, we can find one, say $d_{n}$, in $D$.

Now we come to the question how to make sure that our convex compact sets are simplices. The Urysohn space has the extension property saying that every Katětov function defined on its finite subset is realized as a distance function to some point in the space. Huhunaišvili [42] (cf also [9,36,45,59, 60]) showed that the same is true for Katětov functions defined on compact subsets of the Urysohn space. This is probably the strongest result that can guarantee that certain Katětov functions can be realized in the Urysohn space (or the Urysohn sphere). The following construction is motivated by the need of realizing Katětov functions defined on non-compact subspaces of the Urysohn sphere.

Let $\left(X, d_{X}\right)$ be a separable metric space of diameter bounded by 1 and let $D \subseteq X$ be its dense countable subset. Let $R(D)$ be the ring of functions generated by the distance functions $x \mapsto d_{X}(x, d)$ for $d \in D$ and all rational constant functions. Note that $R(D)$ is countable and all functions in $R(D)$ are Lipschitz. Let $R_{1}(D)$ be the family of functions in $R(D)$ which are Lipschitz 1 and have the range contained in $\left[\frac{1}{2}, 1\right]$. Note that dividing a bounded Lipschitz function by an appropriately large constant, we get a Lipschitz 1 function with the range contained in $\left[-\frac{1}{4}, \frac{1}{4}\right]$. Next, adding $\frac{3}{4}$ we get a Lipschitz 1 function with $\operatorname{rng}(f) \subseteq\left[\frac{1}{2}, 1\right]$. This shows that $R_{1}(D)$ is linearly dense in $R(D)$. On the other hand, since $d_{X}$ is bounded by 1 , every function in $R_{1}(D)$ is a Katětov 
function. Recall that the elements of $X$ are identified with Katětov functions on $X$ by the Kuratowski construction [33, Chapter 1.2], i.e. $x \in X$ is identified with the function $y \mapsto d_{X}(x, y)$. Write $F(X)$ for the family of all finitely supported (see [33, Definition 1.2.2]) Katětov functions on $X$ with values in $[0,1]$. Let $E\left(X, d_{X}, D\right)$ be the completion of the space $F(X) \cup R_{1}(D)$ with the sup metric. Note that $E\left(X, d_{X}, D\right.$ ) is an extension of $X$ (as $F(X)$ contains all functions $y \mapsto d_{X}(x, y)$ for $\left.x \in X\right)$, is separable and realizes all finitely supported Katětov functions on $X$. However, it is slightly bigger than the usual one-step Katětov extension since we also have realized the functions in $R_{1}(D)$. A standard argument shows that if $\varphi: X \rightarrow X$ is an isometry, then $\varphi$ extends to an isometry $\varphi^{\prime}$ of $E\left(X, d_{X}, D\right)$ and the definition of $E\left(X, d_{X}, D\right)$ does not depend on the choice of the dense set $D$. Thus, slightly abusing notation, we write $E\left(X, d_{X}\right)$ for $E\left(X, d_{X}, D\right)$. Note, however, that given $D$, we have a canonical countable dense set $D^{E}$ in $E\left(X, d_{X}, D\right)$ consisting of $R_{1}(D)$ and all Katětov functions finitely supported on a subset of $D$ and assuming rational values on their support.

Now, similarly as in the Katětov construction of the Urysohn space, we iterate the above extension construction infinitely many times.

Definition 5.16 Given a separable metric space $\left(X, d_{X}\right)$ and its countable dense subset $D \subseteq X$ define inductively $E^{n}\left(X, d_{X}, D\right)$ and $D^{n}\left(X, d_{X}, D\right) \subseteq$ $E^{n}\left(X, d_{X}, D\right)$ as follows. $E^{0}\left(X, d_{X}, D\right)=\left(X, d_{X}\right)$ and $D_{0}\left(X, d_{X}, D\right)=D$. Given $E^{n}\left(X, d_{X}, D\right)$ and $D^{n}\left(X, d_{X}, D\right)$, which is a countable dense subset of $E^{n}\left(X, d_{X}, D\right)$ let $E^{n+1}\left(X, d_{X}, D\right)=E\left(E^{n}\left(X, d_{X}, D\right), D^{n}\left(X, d_{X}, D\right)\right)$ and let $D^{n+1}\left(X, d_{X}, D\right)$ be $D^{n}\left(X, d_{X}, D\right)^{E}$ (cf the remarks proceeding this definition). Write $E^{\infty}\left(X, d_{X}, D\right)$ for the completion of the space $\bigcup E^{n}\left(X, d_{X}, D\right)$ and $D^{\infty}\left(X, d_{X}, D\right)$ for $\bigcup_{n} D^{n}\left(X, d_{X}, D\right)$.

Again, the construction of $E^{\infty}\left(X, d_{X}, D\right)$ does not depend on the initial choice of the countable dense set $D$ and we will abuse notation writing $E^{\infty}\left(X, d_{X}\right)$ for $E^{\infty}\left(X, d_{X}, D\right)$, unless this can cause confusion. Note that the space $E^{\infty}\left(X, d_{X}\right)$ is actually isometric to the Urysohn sphere since it realizes all finitely supported Katětov functions with values in $[0,1]$. Note also that if $\varphi: X \rightarrow X$ is an isometry, then $\varphi$ extends canonically to an isometry $\varphi^{\infty}: E^{\infty}\left(X, d_{X}\right) \rightarrow E^{\infty}\left(X, d_{X}\right)$. This follows in the same way as the analogous extension property is proved for the Katětov extensions [61, Page 115] from the (above mentioned) fact that $\varphi$ extends to $E\left(X, d_{X}\right)$.

Given a metric space $\left(X, d_{X}\right)$ and its countable dense subset $D$, write $d_{E^{\infty}\left(X, d_{X}\right)} D^{\infty}\left(X, d_{X}\right)$ for the family of functions on $X$ of the form $x \mapsto$ $d_{E^{\infty}\left(X, d_{X}\right)}(x, d)$ for $d \in D^{\infty}\left(X, d_{X}, D\right)$. Write also $R^{\infty}\left(X, d_{X}, D\right)$ for the ring of functions on $X$ generated by $d_{E^{\infty}}\left(X, d_{X}\right) D^{\infty}\left(X, d_{X}\right)$. The next proposition follows rather immediately from the construction. 
Proposition 5.17 Let $\left(X, d_{X}\right)$ be a separable metric space of diameter bounded by 1 and let $D \subseteq X$ be a countable dense set. Then $R^{\infty}\left(X, d_{X}, D\right)$ is contained in the linear span of $d_{E^{\infty}\left(X, d_{X}\right)} D^{\infty}\left(X, d_{X}\right)$.

Proof Write $D^{\infty}$ for $D^{\infty}\left(X, d_{X}, d\right)$ and $d D^{\infty}$ for $d_{E^{\infty}\left(X, d_{X}\right)} D^{\infty}\left(X, d_{X}\right)$. Every function $f$ in $R^{\infty}\left(X, d_{X}, D\right)$ is a polynomial in finitely many functions in $d D^{\infty}$, which in turn are the distance functions to finitely many points in $D^{\infty}$. Thus, there is $n \in \mathbb{N}$ such that $f$ belongs to the ring generated by the distance functions to $D^{n}\left(X, d_{X}, D\right)$. Then, $f$ belongs to the linear span of distance functions to the points in $D^{n+1}\left(X, d_{X}, D\right)$.

Now, fix a countable dense set $D \subseteq \mathbb{U}_{1}$ and write $D^{\infty}$ for $D^{\infty}\left(\mathbb{U}_{1}, d_{\mathbb{U}_{1}}, D\right)$ and $d_{E}$ for the metric on $E^{\infty}\left(\mathbb{U}_{1}, d_{\mathbb{U}_{1}}\right)$. Consider the convex compact set $S\left(E^{\infty}\left(\mathbb{U}_{1}, d_{\mathbb{U}_{1}}\right), d_{E} D^{\infty}\left(\mathbb{U}_{1}, d_{\mathbb{U}_{1}}, D\right)\right)$ and write $S\left(\mathbb{U}_{1}, d_{\mathbb{U}_{1}}\right)$ for the closed convex hull of $\mathbb{U}_{1}$ in $S\left(E^{\infty}\left(\mathbb{U}_{1}, d_{\mathbb{U}_{1}}\right), d_{E} D^{\infty}\left(\mathbb{U}_{1}, d_{\mathbb{U}_{1}}, D\right)\right)$. Note that $S\left(\mathbb{U}_{1}, d_{\mathbb{U}_{1}}\right)$ is equal to $S\left(\mathbb{U}_{1}, d_{\mathbb{U}_{1}}, d_{E} D^{\infty}\right)$. Note also that by the remarks following Definition 5.3 and the fact that $E^{\infty}\left(\mathbb{U}_{1}, d_{\mathbb{U}_{1}}\right)$ is isometric to the Urysohn sphere, the family $d_{E} D^{\infty}$ is saturated.

Recall (Proposition 3.2) that given a subspace $\left(X, d_{X}\right)$ of $\mathbb{U}_{1}$ we write $Z\left(X, d_{X}\right)$ for an isometric copy of $\left(X, d_{X}\right)$ appropriately embedded into $\mathbb{U}_{1}$.

Definition 5.18 For a subspace $\left(X, d_{X}\right)$ of the Urysohn sphere $\left(\mathbb{U}_{1}, d_{\mathbb{U}_{1}}\right)$, write $S_{Z}\left(X, d_{X}\right)$ for $S\left(Z\left(X, d_{X}\right), d_{E} D^{\infty}\right)$.

Note that by Proposition 5.11 and the remarks proceeding Proposition 5.17, up to affine homeomorphism, the above definition does not depend on the choice of the dense countable set $D \subseteq \mathbb{U}_{1}$.

Similarly as with Proposition 5.15, the following result is stated for the Urysohn sphere but holds true for the Urysohn space as well.

Proposition 5.19 The convex compact set $S\left(\mathbb{U}_{1}, d_{\mathbb{U}_{1}}\right)$ is a simplex.

Proof Write $S$ for $S\left(\mathbb{U}_{1}, d_{\mathbb{U}_{1}}\right)$ and $d_{S}$ for the metric on $S$ (induced from the Hilbert cube). Recall that we have fixed a countable dense set $D \subseteq \mathbb{U}_{1}$ and write $d_{E}$ for the metric on $E^{\infty}\left(\mathbb{U}_{1}, d_{\mathbb{U}_{1}}, D\right)$.

Suppose $\mu$ and $v$ are two distinct Borel probability measures in $P(S)$ which are supported on $\operatorname{ext}(S)$. Since as $\mathbb{U}_{1} \subseteq \operatorname{ext}(S)$ is dense by Corollary 5.8, we can pick two sequences of Borel probability measures $\mu_{n} \rightarrow \mu$ and $v_{n} \rightarrow v$ such that $\mu_{n}$ and $v_{n}$ are finitely supported on $\mathbb{U}_{1}$.

Since $\mu$ and $v$ are distinct as elements of $P(S)$, and the coordinate functions (from $[0,1]^{\mathbb{N}}$ ) separate points in $S$, by the Stone-Weierstrass theorem, there is a function $f \in C(S)$ which belongs to the ring generated by the coordinate functions and is such that $\int f d \mu \neq \int f d \nu$. Note that the restrictions of the coordinate functions to $\mathbb{U}_{1}$ are equal to the $d_{E}$-distance functions to the points in $D^{\infty}$. By Proposition 5.17 and the fact that $\mathbb{U}_{1}$ is dense in $\operatorname{ext}(S)$, we can 
assume that the restriction of $f$ to $\mathbb{U}_{1}$ is actually equal to the $d_{E}$-distance function to, say, $z \in D^{\infty}$. Thus, we assume that the function $f$ on $\operatorname{ext}(S)$ is just one of the coordinate functions. Say it is the $k$ th coordinate, i.e. $z$ is the $k$ th element of $D^{\infty}$.

Write $x$ for the barycenter of $\mu$ and $y$ for the barycenter of $v$ and let $x_{n}$ be the barycenter of $\mu_{n}$ and $y_{n}$ of $v_{n}$. Note that $x_{n} \rightarrow x$ and $y_{n} \rightarrow y$ [44, Chapter 15, Proposition 2.2]. Now, since $\mu_{n}$ and $v_{n}$ are supported on $\mathbb{U}_{1}$, similarly as in Proposition 5.9, we get that $x_{n}(k)=\int f d \mu_{n}$ and $y_{n}(k)=$ $\int f d \nu_{n}$. But $\int f d \mu_{n} \rightarrow \int f d \mu$ and $\int f d \nu_{n} \rightarrow \int f d \nu$. Thus, $x(k)=\int f d \mu$ and $y(k)=\int f d v$ and hence $x$ and $y$ are distinct, as needed. This ends the proof.

Corollary 5.20 For every closed subspace $\left(X, d_{X}\right)$ of $\mathbb{U}_{1}$ the set $S_{Z}\left(X, d_{X}\right)$ is a simplex and $Z\left(X, d_{X}\right)$ is a dense subset of $\operatorname{ext}\left(S_{Z}\left(X, d_{X}\right)\right)$.

Proof For simplicity identify $\left(X, d_{X}\right)$ with $Z\left(X, d_{X}\right)$. Note that $S_{Z}\left(X, d_{X}\right)$ is equal to the closed convex hull of $X$ in $S\left(\mathbb{U}_{1}, d_{\mathbb{U}_{1}}\right)$ and $X$ is contained in $\operatorname{ext}\left(S\left(\mathbb{U}_{1}, d_{\mathbb{U}_{1}}\right)\right)$. Thus, $S_{Z}\left(X, d_{X}\right)$ is a face of $S\left(\mathbb{U}_{1}, d_{\mathbb{U}_{1}}\right)$. By Proposition 5.19, this implies [44, Chapter 15, Corollary 3.3] that $S_{Z}\left(X, d_{X}\right)$ is a simplex. Clearly, $X$ is contained in $\operatorname{ext}\left(S_{Z}\left(X, d_{X}\right)\right)$. The fact that $X$ is dense in $\operatorname{ext}\left(S_{Z}\left(X, d_{X}\right)\right)$ follows directly from Corollary 5.8.

Finally, we need to see that $S$-extensions of metric spaces are invariant under the isometry of the metric spaces.

Proposition 5.21 Suppose $\left(X, d_{X}\right)$ and $\left(Y, d_{Y}\right)$ subspaces of $\mathbb{U}_{1}$. If $\left(X, d_{X}\right)$ and $\left(Y, d_{Y}\right)$ are isometric, then the simplices $S_{Z}\left(X, d_{X}\right)$ and $S_{Z}\left(Y, d_{Y}\right)$ are affinely homeomorphic via a map that extends the isometry of $Z\left(X, d_{X}\right)$ and $Z\left(Y, d_{Y}\right)$.

Proof For simplicity again identify $\left(X, d_{X}\right)$ with $Z\left(X, d_{X}\right)$ and $\left(Y, d_{Y}\right)$ with $Z\left(Y, d_{Y}\right)$. Let $\varphi: \mathbb{U}_{1} \rightarrow \mathbb{U}_{1}$ be an isometry such that $\varphi^{\prime \prime} X=Y$. Let $D \subseteq \mathbb{U}_{1}$ be the fixed countable dense set and let $E=\varphi^{\prime \prime} D$. Write $\varphi^{\infty}: E^{\infty}\left(\mathbb{U}_{1}, d_{\mathbb{U}_{1}}\right) \rightarrow$ $E^{\infty}\left(\mathbb{U}_{1}, d_{\mathbb{U}_{1}}\right)$ for the extension of $\varphi$ and note that $\left(\varphi^{\infty}\right)^{\prime \prime} D^{\infty}=E^{\infty}$. Write $d$ for the metric on $E^{\infty}\left(\mathbb{U}_{1}, d_{\mathbb{U}_{1}}\right)$. Note that since $\varphi^{\infty}$ is an isometry, the sets $S\left(E^{\infty}\left(\mathbb{U}_{1}, d_{\mathbb{U}_{1}}\right), D^{\infty}, d D^{\infty}\right)$ and $S\left(E^{\infty}\left(\mathbb{U}_{1}, d_{\mathbb{U}_{1}}\right), E^{\infty}, d E^{\infty}\right)$ are equal and we get the following diagram:

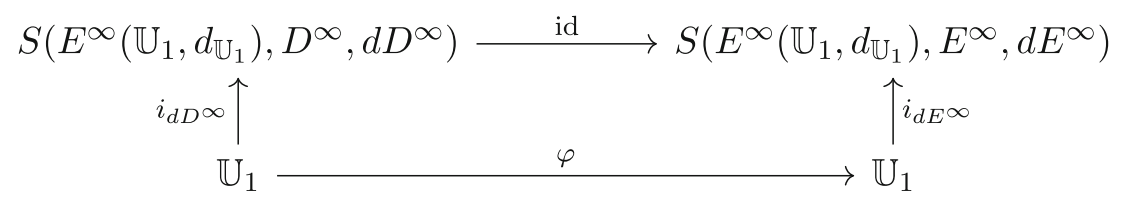

Composing this with the diagram in Proposition 5.11 (where we take the restrictions of the maps $i_{d D^{\infty}}$ and $i_{d E^{\infty}}$ to $\mathbb{U}_{1}$ ), we get 


$$
\begin{aligned}
S\left(E^{\infty}\left(\mathbb{U}_{1}, d_{\mathbb{U}_{1}}\right), D^{\infty}, d D^{\infty}\right) & \underset{D^{\infty}}{E^{\infty}} \\
i_{d D^{\infty} \uparrow} & S\left(E^{\infty}\left(\mathbb{U}_{1}, d_{\mathbb{U}_{1}}\right), D^{\infty}, d D^{\infty}\right) \\
\mathbb{U}_{1} \longrightarrow i_{d D^{\infty}} & \mathbb{U}_{1}
\end{aligned}
$$

and

since all the maps are affine and continuous, this immediately implies that $\tau_{D^{\infty}}^{E^{\infty}} \operatorname{maps} \operatorname{conv}\left(i_{d D^{\infty}} X\right)=S_{Z}\left(X, d_{X}\right)$ to $\operatorname{conv}\left(i_{d D^{\infty}} Y\right)=S_{Z}\left(Y, d_{Y}\right)$.

The simplex $S\left(\mathbb{U}_{1}, d_{\mathbb{U}_{1}}\right)$ plays now the role of a universal homogeneous Choquet simplex. However, it does not seem to be affinely homeomorphic to the Poulsen simplex. On the other hand, that there is another convex compact set, which seems to be related to the Poulsen simplex. It is the set $S\left(\mathbb{U}_{1}, d_{\mathbb{U}_{1}}, D, d_{\mathbb{U}_{1}} D\right)$. Write $S^{\prime}\left(\mathbb{U}_{1}, d_{\mathbb{U}_{1}}\right)$ for $S\left(\mathbb{U}_{1}, d_{\mathbb{U}_{1}}, D, d_{\mathbb{U}_{1}} D\right)$ (for a countable dense set $\left.D \subseteq \mathbb{U}_{1}\right)$. Again, up to affine homeomorphism, it does not depend on the choice of the countable set $D$. Now, the map from $S\left(\mathbb{U}_{1}, d_{\mathbb{U}_{1}}\right)$ to $S^{\prime}\left(\mathbb{U}_{1}, d_{\mathbb{U}_{1}}\right)$, which forgets about the coordinates corresponding to $D^{\infty} \backslash D$ is clearly affine and continuous and hence $S\left(\mathbb{U}_{1}, d_{\mathbb{U}_{1}}\right)$ can be treated as an unfolded version of $S^{\prime}\left(\mathbb{U}_{1}, d_{\mathbb{U}_{1}}\right)$. We do not know if $S^{\prime}\left(\mathbb{U}_{1}, d_{\mathbb{U}_{1}}\right)$ is a simplex but it seems to be more closely related to the Pousen simplex than $S\left(\mathbb{U}_{1}, d_{\mathbb{U}_{1}}\right)$.

Proposition $5.22 S^{\prime}\left(\mathbb{U}_{1}, d_{\mathbb{U}_{1}}\right)$ has a dense set of extreme points.

The proof follows from the following simple claim.

Claim 5.23 If $g$ and $h$ are Katětov functions and $\alpha \in[0,1]$, then $\alpha f+(1-\alpha) g$ is also a Katětov function.

Proof This is an elementary computation. The Katětov inequalities for $\alpha f+$ $(1-\alpha) g$ follow directly from the Katětov inequalities for $f$ and $g$.

Proof of Proposition 5.22 By the fact that $\mathbb{U}_{1} \subseteq \operatorname{ext}\left(S^{\prime}\left(\mathbb{U}_{1}, d_{\mathbb{U}_{1}}\right)\right)$, it is enough to check that $\mathbb{U}_{1}$ is dense in $S^{\prime}\left(\mathbb{U}_{1}, d_{\mathbb{U}_{1}}\right)$. Let $s \in S^{\prime}\left(\mathbb{U}_{1}, d_{\mathbb{U}_{1}}\right)$ and $\varepsilon>0$. Find $n$ such that $2^{-(n+1)}<\varepsilon / 2$ and $s$ is $\varepsilon$-close to $S_{n}^{n}\left(\mathbb{U}_{1}, d_{\mathbb{U}_{1}}, D, d_{\mathbb{U}_{1}} D\right)$. Write $D=\left(d_{1}, d_{2}, \ldots\right)$ and $\mathbb{U}_{1}\left\lceil n\right.$ for the image of $\mathbb{U}_{1} \subseteq S\left(\mathbb{U}_{1}, d_{\mathbb{U}_{1}}, D, d_{\mathbb{U}_{1}} D\right)$ under the projection map from $[0,1]^{\mathbb{N}}$ to $[0,1]^{n}$. Note that it suffices to show that $S_{n}^{n}\left(\mathbb{U}_{1}, d_{\mathbb{U}_{1}}, D, d_{\mathbb{U}_{1}} D\right)$ is contained in $\mathbb{U}_{1}\lceil n$. Clearly, the vertices of the simplex $S_{n}^{n}\left(\mathbb{U}_{1}, d_{\mathbb{U}_{1}}, D, d_{\mathbb{U}_{1}} D\right)$ belong to $\mathbb{U}_{1}\left\lceil n\right.$. For each $i \leq n$ let $f_{i}:\left\{d_{1}, \ldots, d_{n}\right\} \rightarrow$ $[0,1]$ be defined $f_{i}(z)=d\left(d_{i}, z\right)$. Now, Claim 5.23 implies that for every $\alpha_{1}, \ldots, \alpha_{n} \in[0,1]$ with $\sum_{i} \alpha_{i}=1$ the function $\sum_{i} \alpha_{i} f_{i}$ is a Katětov function with values in $[0,1]$, thus realized in $\mathbb{U}_{1}$. This implies that the set of points $\left(d\left(x, d_{1}\right), \ldots, d\left(x, d_{n}\right)\right) \in[0,1]^{n}$ for $x \in \mathbb{U}_{1}$ is convex. But the latter set is equal to $\mathbb{U}_{1}\left\lceil n\right.$. Thus, since the vertices of $S_{n}^{n}\left(\mathbb{U}_{1}, d_{\mathbb{U}_{1}}, D, d_{\mathbb{U}_{1}} D\right)$ belong to $\mathbb{U}_{1}\left\lceil n\right.$ and $\mathbb{U}_{1}\left\lceil n\right.$ is convex, we have that $S_{n}^{n}\left(\mathbb{U}_{1}, d_{\mathbb{U}_{1}}, D, d_{\mathbb{U}_{1}} D\right)$ is contained in $\mathbb{U}_{1}\lceil n$, as needed. This ends the proof. 


\section{Iterated cone construction}

Given a simplex $S$ and a point $s \in S$ we define the cone of $S$ over $s$ as follows. Consider $Y=S \times[0,1]$ and let

$$
\operatorname{cone}(S, s)=\operatorname{conv}((S \times\{0\}) \cup(s, 1)),
$$

where the convex hull is taken in $Y$ (recall our convention on metrics in Sect. 2 and note that if $S$ is a subset of the Hilbert cube, then the cone is embedded in the Hilbert cube as well).

The point $(s, 1)$ is called the cone point of the cone and we will identify $S$ with $S \times\{0\} \subseteq$ cone $(S, s)$. We also denote the cone point as $c(s)$. The cone admits a natural affine continuous map $\pi: \operatorname{cone}(S, s) \rightarrow S$ which is just the projection map in $Y$. It maps the cone point $c(s)$ to the point $s$.

We state now a couple of basic facts on the structure of cones.

Lemma 6.1 If $S$ is a simplex and $s \in S$, then $\operatorname{cone}(S, s)$ is a simplex, $S$ is a face of cone $(S, s)$ and $\operatorname{ext}(\operatorname{cone}(S, s))=\operatorname{ext}(S) \cup\{c(s)\}$.

Proof Note that if two points $z_{1}, z_{2} \in S \times[0,1]$ have an affine combination that lies in $S$, then both $z_{1}$ and $z_{2}$ must belong to $S$. This implies that $S$ is a face of the convex compact set cone $(S, s)$ and that $\operatorname{ext}(S) \subseteq \operatorname{ext}(\operatorname{cone}(S, s))$. It is clear that $c(s)$ is an extreme point of cone $(S, s)$, so indeed $\operatorname{ext}(\operatorname{cone}(S, s))=$ $\operatorname{ext}(S) \cup\{c(s)\}$. To see that cone $(S, s)$ is a simplex note that if $\mu$ is a measure concentrated on $\operatorname{ext}(S) \cup\{c(s)\}$ such that $\int f d \mu=0$ for every affine continuous function on cone $(S, s)$, then $\mu(\{c(s)\})=0$ and hence $\mu$ must be concentrated on $\operatorname{ext}(S)$.

Lemma 6.2 Given a simplex $S$ and two points $s_{1}, s_{2} \in S$ we have

$$
\text { cone }\left(\operatorname{cone}\left(S, s_{1}\right), s_{2}\right) \simeq \operatorname{cone}\left(\operatorname{cone}\left(S, s_{2}\right), s_{1}\right) \text {. }
$$

Proof Both simplices are affinely homeomorphic to the closed convex hull of $S \times\{(0,0)\},\left(s_{1}, 0,1\right)$ and $\left(s_{2}, 1,0\right)$ in $S \times[0,1]^{2}$. To see the affine homeomorphism of cone (cone $\left.\left(S, s_{1}\right), s_{2}\right)$ and cone (cone $\left.\left(S, s_{2}\right), s_{1}\right)$ directly, write $c\left(s_{1}\right)$ for the cone point of cone $\left(S, s_{1}\right), c^{\prime}\left(s_{2}\right)$ for the cone point of cone (cone $\left.\left(S, s_{1}\right), s_{2}\right), c\left(s_{2}\right)$ for the cone point of cone $\left(S, s_{2}\right)$ and $c^{\prime}\left(s_{1}\right)$ for the cone point of cone $\left.\left(\operatorname{cone}\left(S, s_{2}\right), s_{1}\right)\right)$. The affine homeomorphism then maps $(1-\beta)\left((1-\alpha) x+\alpha c\left(s_{1}\right)\right)+\beta c^{\prime}\left(s_{2}\right)$ to $(1-\gamma)\left((1-\delta) x+\delta c\left(s_{2}\right)\right)+\gamma c^{\prime}\left(s_{1}\right)$, where $\gamma=(1-\beta) \alpha$ and $\delta=\beta /(1-\alpha(1-\beta))$.

Given the above lemma, we use the notation cone $\left(S, s_{1}, \ldots, s_{n}\right)$ to denote an iterated cone of the form cone $\left(\ldots \operatorname{cone}\left(S, s_{1}\right) \ldots, s_{n}\right)$ (or with any other permutation). We also call a cone of the form cone $\left(S, s_{1}, s_{2}\right)$ a double cone. 
Given a subset $\left\{i_{1}, \ldots, i_{k}\right\} \subseteq\{1, \ldots, n\}$, the simplex cone $\left(S, s_{i_{1}}, \ldots, s_{i_{k}}\right)$ is a subset of cone $\left(S, s_{1}, \ldots, s_{n}\right)$ in the same way $S$ is a subset of cone $(S, s)$. The simplex cone $\left(S, s_{i_{1}}, \ldots, s_{i_{k}}\right)$ is then a face of $\operatorname{cone}\left(S, s_{1}, \ldots, s_{n}\right)$. We call a face of the form cone $\left(S, s_{i_{1}}, \ldots, s_{i_{k}}\right)$ in cone $\left(S, s_{1}, \ldots, s_{n}\right)$ a subcone.

Lemma 6.3 Given a simplex $S$ and $s_{1}, \ldots, s_{n} \in S$, if $\left\{i_{1}, \ldots, i_{k}\right\} \subseteq\{1, \ldots, n\}$ and $\left\{j_{1}, \ldots, j_{n-k}\right\}=\{1, \ldots, n\} \backslash\left\{i_{1}, \ldots, i_{k}\right\}$, then

$$
\operatorname{cone}\left(S, s_{1}, \ldots, s_{n}\right) \simeq \operatorname{cone}\left(\operatorname{cone}\left(S, s_{i_{1}}, \ldots, s_{i_{k}}\right), s_{j_{1}}, \ldots, s_{j_{n-k}}\right) .
$$

Proof This follows directly from the definitions and Lemma 6.2.

Definition 6.4 Given a simplex $S$ and its countable (enumerated) subset $D=$ $\left(d_{n}: n \in \mathbb{N}\right)$, define the iterated cone over $D$ as follows. Let $S_{0}=S$ and for each $n \in \mathbb{N}$ let $S_{n}=\operatorname{cone}\left(S_{n-1}, d_{n}\right)$ and let $\pi_{n}: S_{n+1} \rightarrow S_{n}$ be the projection map. Define

$$
\text { cone }(S, D)=\lim _{\longleftarrow}\left(S_{n}, \pi_{n}\right) .
$$

Note that if $S$ is a subset of the Hilbert cube, then cone $(S, D)$ can be naturally embedded into the Hilbert cube as well. Note also that the inverse system in the above definition is increasing. Thus, we treat $S_{n}$ as a face of cone $(S, D)$, for each $n \in \mathbb{N}$. Given that, all cone points of the simplices $S_{n}$ belong to cone $(S, D)$ and we refer to them as to the cone points of the iterated cone. In principle, the iterated cone over $D$ may depend on the ordering of the set $D$. As we will see, this does not happen.

Lemma 6.5 Let $S$ be a simplex, $\varepsilon>0$ and $\varphi: S \rightarrow S$ be an affine homeomorphism. Given two points $s_{1}, s_{2} \in S$ if $d_{S}\left(\varphi\left(s_{1}\right), s_{2}\right)<\varepsilon$, then there is an affine homeomorphism $\varphi^{\prime}: \operatorname{cone}\left(S, s_{1}\right) \rightarrow \operatorname{cone}\left(S, s_{2}\right)$ extending $\varphi$ such that

$$
d_{S}\left(\varphi\left(\pi_{1}(s)\right), \pi_{2}\left(\varphi^{\prime}(s)\right)\right)<\varepsilon
$$

for every $s \in \operatorname{cone}\left(S, s_{1}\right)$, where $\pi_{1}: \operatorname{cone}\left(S, s_{1}\right) \rightarrow S$ and $\pi_{2}:$ cone $\left(S, s_{2}\right) \rightarrow S$ are the projection maps.

Proof The map $\varphi^{\prime}$ is the affine map whose restriction to $S$ is $\varphi$ and which maps $c\left(s_{1}\right)$ to $c\left(s_{2}\right)$. To see that $d_{S}\left(\pi_{2}\left(\varphi^{\prime}(s)\right), \varphi\left(\pi_{1}(s)\right)\right)<\varepsilon$ for each $s \in$ cone $\left(S, s_{1}\right)$, note that $\pi_{2} \circ \varphi^{\prime}$ and $\varphi \circ \pi_{1}$ are two affine continuous maps such that $d_{S}\left(\pi_{2}\left(\varphi^{\prime}(e)\right), \varphi\left(\pi_{1}(e)\right)\right)<\varepsilon$ holds for every extreme point of cone $\left(S, s_{1}\right)$. Thus, by Proposition 2.1, $d_{S}\left(\pi_{2}\left(\varphi^{\prime}(s)\right), \varphi\left(\pi_{1}(s)\right)\right)<\varepsilon$ is true for every $s \in$ cone $\left(S, s_{1}\right)$.

Proposition 6.6 Given a simplex $S$ and an affine homeomorphism $\varphi: S \rightarrow$ $S$, if $D=\left(d_{n}: n \in \mathbb{N}\right)$ and $E=\left(e_{n}: n \in \mathbb{N}\right)$ are two subsets of $S$ 
such that $\operatorname{cl}(E)=\operatorname{cl}\left(\varphi^{\prime \prime} D\right)$, then there is an extension $\varphi^{\prime}$ of $\varphi$ to an affine homeomorphism $\varphi^{\prime}: \operatorname{cone}(S, D) \rightarrow \operatorname{cone}(S, E)$.

Proof Write $X=\operatorname{cone}(S, D)$ and $Y=\operatorname{cone}(S, E)$ and define $X_{n}$ and $Y_{n}$ as $X_{0}=Y_{0}=S$ and $X_{n+1}=\operatorname{cone}\left(X_{n}, d_{n}\right)$ and $Y_{n+1}=\operatorname{cone}\left(Y_{n}, e_{n}\right)$ so that $X=\lim X_{n}$ and $Y=\lim _{\leftarrow} Y_{n}$. We define inductively two increasing sequences of natural numbers $n_{i} \overleftarrow{\text { and }} m_{i}$ and affine continuous maps $\varphi_{i}: X_{n_{i}} \rightarrow Y_{m_{i}}$ and $\psi_{i}: Y_{m_{i}} \rightarrow X_{n_{i+1}}$ such that $\varphi_{0}=\varphi$ and

- $\left(\varphi_{n}, \psi_{n}: n \in \mathbb{N}\right)$ is an approximate intertwining.

- $\operatorname{rng}\left(\varphi_{n}\right)$ and $\operatorname{rng}\left(\psi_{n}\right)$ is a subcone of $X$ or $Y$, for each $n \in \mathbb{N}$.

The theorem will then follow by Proposition 4.1.

Let $\varphi_{0}=\psi_{0}^{-1}=\varphi$. Suppose $\varphi_{i}, \psi_{i}$ are defined. We need to define $\varphi_{i+1}$ : $X_{n_{i+1}} \rightarrow Y_{m_{i+1}}$. Let $\varphi_{i}^{\prime}=\psi_{i}^{-1}$ and $X_{n_{i}}^{\prime}=\operatorname{rng}\left(\psi_{i}\right)=\operatorname{dom}\left(\varphi_{i}^{\prime}\right)$. By our assumption, $X_{n_{i}}^{\prime}$ is a subcone, so there are $p_{1}, \ldots, p_{k} \in \mathbb{N}$ such that $X_{n_{i}}^{\prime}=$ cone $\left(S, d_{p_{1}}, \ldots, d_{p_{k}}\right)$. Find $n_{i+1}>n_{i}$ such that $p_{j}<n_{i+1}$ for each $j \leq$ $k$. Let $\left\{q_{1}, \ldots, q_{l}\right\}=\left\{1, \ldots, n_{i+1}\right\} \backslash\left\{p_{1}, \ldots, p_{k}\right\}$. By Lemma 6.3, $X_{n_{i+1}}$ is affinely homeomorphic to cone $\left(X_{n_{i}}^{\prime}, d_{q_{1}}, \ldots, d_{q_{l}}\right)$. For each $j \leq l$ write $X_{i}^{j}=$ cone $\left(X_{n_{i}}^{\prime}, d_{q_{1}}, \ldots, d_{q_{j}}\right)$. We inductively find increasing numbers $m_{i}^{j} \in \mathbb{N}$ and $\operatorname{maps} \varphi_{i}^{j}: X_{i}^{j} \rightarrow Y_{m_{i}^{j}}$ so that $\varphi_{i}^{0}=\varphi_{i}^{\prime}, \varphi_{i}^{j} \subseteq \varphi_{i}^{j+1}$ and for each $j$ we have

$$
d_{m_{i}^{j}}\left(\varphi _ { i } ^ { j } \left(x\left\lceil X_{n_{i}}\right), \varphi_{i}^{j}(x)\left\lceil Y_{m_{i}}\right)<\frac{1}{2^{i+j}}\right.\right.
$$

for each $x \in X_{i}^{j}$. Given $\varphi_{i}^{j}$, find $m_{i}^{j+1}$ such that

$$
d_{S}\left(\varphi\left(d_{q_{j+1}}\right), e_{m_{i}^{j+1}}\right)=d_{m_{i}^{j}}\left(\varphi_{i}^{j}\left(d_{q_{j+1}}\right), e_{m_{i}^{j+1}}\right)<\frac{1}{2^{i+j}}
$$

and use Lemma 6.5 to extend $\varphi_{i}^{j}$ to $\varphi_{i}^{j+1}$ so that (3) holds. At the end, put $\varphi_{i+1}=\varphi_{i}^{l}$ and $m_{i+1}=m_{i}^{l}$. The map $\psi_{i+1}$ is defined analogously.

The condition (3) and an analogous inequality for $\psi_{i}$ witness that $\varphi_{i}$ and $\psi_{i}$ form an approximate intertwining.

Applying Proposition 6.6 to $\varphi=$ id we get that the definition of cone $(S, D)$ indeed does not depend on the ordering of $D$.

Lemma 6.7 Given a simplex $S$, with a countable subset $D$ and $d \in S \backslash D$ we have

$$
\operatorname{cone}(S,\{d\} \cup D) \simeq \operatorname{cone}(\operatorname{cone}(S, D), d) .
$$


Proof Note first that if $\left(X_{n}, \pi_{n}: n \in \mathbb{N}\right)$ is an increasing inverse system of simplices and $x \in X_{0}$, then cone $\left(\lim X_{n}, x\right) \simeq \lim \operatorname{cone}\left(X_{n}, x\right)$, where the latter inverse system has the natural projection maps $\pi_{n}^{\prime}$ : cone $\left(X_{n}, x\right) \rightarrow$ cone $\left(X_{n-1}, x\right)$ that extend $\pi_{n}$ and map the cone point to the cone point.

Write $D=\left\{d_{1}, d_{2}, \ldots\right\}$ and $X_{n}=\operatorname{cone}\left(S, d_{1}, \ldots, d_{n}\right)$. By the remark above, cone $(\operatorname{cone}(S, D), d) \simeq \lim \operatorname{cone}\left(X_{n}, d\right)$ and by Lemma 6.2 we have cone $\left(X_{n}, d\right) \simeq$ cone $\left(S, d, d_{1}, \ldots, d_{n}\right)$. Thus, cone $(\operatorname{cone}(S, D), d)$ is affinely homeomorphic to $\lim _{\leftarrow}\left(\operatorname{cone}\left(S, d, d_{1}, \ldots, d_{n}\right)\right)$, which is equal to cone $(S,\{d\} \cup$ $D)$.

Now we look at the extreme point of the iterated cones.

Lemma 6.8 Suppose $S$ is a simplex, $s_{1}, s_{2} \in S$ and let $y \in \operatorname{cone}\left(S, s_{1}\right)$ be such that

$$
y=\alpha x+(1-\alpha) c\left(s_{1}\right)
$$

for some $x \in S$ and $0<\alpha<1$. Write $\pi: \operatorname{cone}\left(\operatorname{cone}\left(S, s_{1}\right), s_{2}\right) \rightarrow$ cone $\left(S, s_{1}\right)$ for the projection map. Then for every $y^{\prime} \in \operatorname{cone}\left(\operatorname{cone}\left(S, s_{1}\right), s_{2}\right)$ with $\pi\left(y^{\prime}\right)=y$ there exists a unique $x^{\prime} \in \operatorname{cone}\left(S, s_{2}\right)$ such that

$$
y^{\prime}=\alpha x^{\prime}+(1-\alpha) c\left(s_{1}\right),
$$

and $\pi\left(x^{\prime}\right)=x$.

Proof Uniqueness of $x^{\prime}$ is immediate. Let $c_{1}=c\left(s_{1}\right)$ and $c_{2}=c\left(s_{2}\right)$. As $y^{\prime} \in$ cone (cone $\left.\left(S, s_{1}\right), s_{2}\right)$, there is $z \in \operatorname{cone}\left(S, s_{1}\right)$ and $\delta \in[0,1]$ such that $y^{\prime}=\delta z+(1-\delta) c_{2}$. As $z \in \operatorname{cone}\left(S, s_{1}\right)$, there is $w \in S$ and $\rho \in[0,1]$ such that $z=\rho w+(1-\rho) c_{1}$.

Note that $0<\alpha$ implies $y \neq c_{1}$, and so $\delta \rho+1-\delta \neq 0$, for if it were 0 , then $\delta=1, \rho=0$ and so $y^{\prime}=z=c_{1}$, meaning $y=\pi\left(y^{\prime}\right)=\pi\left(c_{1}\right)=c_{1}$. Similarly, $\alpha<1$ implies $y \notin S$, and so $\delta \rho+1-\delta \neq 1$, for if it were 1 , then either $\delta=0$ or $\rho=1$, and in either case $y^{\prime} \in \operatorname{cone}\left(S, s_{2}\right)$, meaning $y=\pi\left(y^{\prime}\right) \in S$.

Note that

$$
\begin{aligned}
y^{\prime} & =\delta z+(1-\delta) c_{2}=\delta \rho w+(\delta-\delta \rho) c_{1}+(1-\delta) c_{2} \\
& =(\delta \rho+1-\delta)\left[\frac{\delta \rho}{\delta \rho+1-\delta} w+\frac{1-\delta}{\delta \rho+1-\delta} c_{2}\right]+(\delta-\delta \rho) c_{1}
\end{aligned}
$$

and put $x^{\prime}=\frac{\delta \rho}{\delta \rho+1-\delta} w+\frac{1-\delta}{\delta \rho+1-\delta} c_{2}$ so that

$$
y^{\prime}=(\delta \rho+1-\delta) x^{\prime}+(\delta-\delta \rho) c_{1} .
$$


Note that $x^{\prime} \in \operatorname{cone}\left(S, s_{2}\right)$. We claim that $\pi\left(x^{\prime}\right)=x$ and $\delta \rho+1-\delta=\alpha$. This will imply that $y^{\prime}=\alpha x^{\prime}+(1-\alpha) c_{2}$, i.e. that $x^{\prime}$ is as needed.

First, $\pi\left(x^{\prime}\right)=\frac{\delta \rho}{\delta \rho+1-\delta} \pi(w)+\frac{1-\delta}{\delta \rho+1-\delta} \pi\left(c_{2}\right)$. But $\pi(w)=w \in S$ and $\pi\left(c_{2}\right)=s_{2}$, so indeed $\pi\left(x^{\prime}\right) \in S$. Now,

$$
y=\pi\left(y^{\prime}\right)=(\delta \rho+1-\delta) \pi\left(x^{\prime}\right)+(\delta-\delta \rho) \pi\left(c_{1}\right)
$$

and $\pi\left(c_{1}\right)=c_{1}$. As every element of cone $\left(S, s_{1}\right) \backslash\left(S \cup\left\{c_{1}\right\}\right)$ is uniquely determined as a convex combination of $c_{1}$ and an element of $S$, we necessarily have $\pi\left(x^{\prime}\right)=x$ and $\delta \rho+1-\delta=\alpha$.

Lemma 6.9 Given a simplex $S$ and its countable subset $D$ let $\left\{v_{n}: n \in \mathbb{N}\right\}$ be the set of cone points of cone $(S, D)$. Then

$$
\operatorname{ext}(\operatorname{cone}(S, D))=\operatorname{ext}(S) \cup\left\{v_{n}: n \in \mathbb{N}\right\} .
$$

Proof By Lemma 6.1, $S$ is a face of cone $(S, D)$, so ext $(S) \subseteq \operatorname{ext}(\operatorname{cone}(S, D))$. Also, for each $n$ the simplex cone $\left(S, d_{0}, \ldots, d_{n}\right)$ is a face of cone $(S, D)$, which shows that $v_{n} \in \operatorname{ext}(\operatorname{cone}(S, D))$. We need to show that there are no more extreme points in cone $(S, D)$. Let $y \in \operatorname{cone}(S, D)$ be such that $y \notin S$ and $y \neq v_{n}$ for each $n$. Pick $n$ such that $y\lceil n \notin S$. This means that there exists $\lambda$ with $0<\lambda<1$ and $s \in S$ such that

$$
y\left\lceil n=\lambda s+(1-\lambda) v_{n} .\right.
$$

By Lemma 6.8, for each $m>n$ there exists $s_{m} \in \operatorname{cone}\left(S, d_{n+1}, \ldots, d_{m}\right)$ such that

$$
y\left\lceil m=\lambda s_{m}+(1-\lambda) v_{n} .\right.
$$

Uniqueness of $s_{m}$ implies that $s_{m_{2}}\left\lceil m_{1}=s_{m_{1}}\right.$ for each $m_{2}>m_{1}>n$. Let $x \in \operatorname{cone}\left(S,\left\{d_{n+1}, d_{n+2}, \ldots\right\}\right)$ be such that $x \nmid k=s_{k}$ for each $k>n$. By Lemma 6.7, we have

$$
\operatorname{cone}(S, D)=\operatorname{cone}\left(\operatorname{cone}\left(S,\left\{d_{n+1}, d_{n+2}, \ldots\right\}\right), d_{0}, \ldots, d_{n}\right)
$$

and in the latter simplex we have $y=\lambda x+(1-\lambda) v_{n}$, which shows that $y$ is not an extreme point of cone $(S, D)$.

Lemma 6.10 Given a simplex $S$ and its countable subset $D$, let $\left\{v_{n}: n \in \mathbb{N}\right\}$ be the set of cone points of $\operatorname{cone}(S, D)$. Then each $v_{n}$ is an isolated point of $\operatorname{ext}(\operatorname{cone}(S, D))$. 
Proof Write $D=\left\{d_{1}, d_{2}, \ldots\right\}$ so that $v_{n}$ is the cone point over $d_{n}$, for each $n \in$ $\mathbb{N}$. We need to show that $v_{n}$ is isolated from $\operatorname{ext}(S) \cup\left\{v_{i}: i \neq n\right\}$. Write $\pi_{n}^{\infty}$ : cone $(S, D) \rightarrow$ cone $\left(S, d_{1}, \ldots, d_{n}\right)$ for the projection map. Clearly, $v_{n}=$ $\pi_{n}^{\infty}\left(v_{n}\right)$ has positive distance from $S \cup\left\{v_{i}: i<n\right\}$ in cone $\left(S, d_{1}, \ldots, d_{n}\right)$, so let $U$ be an open neighborhood of $v_{n}$ in cone $\left(S, d_{1}, \ldots, d_{n}\right)$ that is disjoint from $S$ and $\left\{v_{i}: i<n\right\}$. Then $\left(\pi_{n}^{\infty}\right)^{-1}(U)$ is an open neighborhood of $v_{n}$ that is disjoint from $\operatorname{ext}(S)$ and does not contain any $v_{i}$ with $i \neq n$.

\section{The blow-up construction}

Given a countable dense subset $D$ of a metric space $\left(X, \delta_{X}\right)$ with $\delta_{X}<\frac{1}{2}$, enumerate as $\left(p_{n}=\left(x_{n}, y_{n}, s_{n}, t_{n}\right): n \in \mathbb{N}\right)$, with infinite repetitions, all quadruples $(x, y, s, t)$ with $x, y \in D$ distinct and $s<t \in \mathbb{Q} \cap\left(0, \frac{1}{2}\right)$ such that $s<\delta_{X}(x, y)<t$. Call the sequence $\left(p_{n}: n \in \mathbb{N}\right)$ the metric scheme of $\left(X, \delta_{X}, D\right)$ and denote it by $\operatorname{Sch}\left(X, \delta_{X}, D\right)$. For every $n \in \mathbb{N}$ and $p_{n}=$ $\left(x_{n}, y_{n}, s_{n}, t_{n}\right)$ in the metric scheme, write $p_{n}(1)$ for $x_{n}$ and $p_{n}(2)$ for $y_{n}$. Write also $p_{n}(D)$ for the $n$th element of $\operatorname{Sch}\left(X, d_{X}, D\right)$.

Recall that given a simplex $S$ and two points $x_{1}, x_{2} \in S$ the double cone cone $\left(S, x_{1}, x_{2}\right)$ is the simplex cone (cone $\left.\left(S, x_{1}\right), x_{2}\right)$. Now, given a simplex $S$ and its subset $X \subseteq S$ together with a metric $d_{X}$ on $X$ we will define another, bigger, simplex, which will be used to encode the metric in its affine structure.

Let $\left(p_{n}: n \in \mathbb{N}\right)$ enumerate the metric scheme of $\left(X, \frac{1}{2} d_{X}\right)$. Define an increasing inverse system of simplices as follows. Let $B_{0}=S$ and $B_{n}=$ cone $\left(B_{n-1}, p_{n}(1), p_{n}(2)\right)$. Define the blow-up of $S$ with respect to $\left(X, d_{X}\right)$ (slightly abusing the notation), denoted by $B\left(S, X, d_{X}\right)$ as $\lim B_{n}$ and write $c_{1}\left(p_{n}\right)$ for the cone point over $p_{n}(1)$ in $B\left(S, X, d_{X}\right)$ and $c_{2}\left(\overleftarrow{p_{n}}\right)$ for the cone point over $p_{n}(2)$ in $B\left(S, X, d_{X}\right)$.

Definition 7.1 Given a closed subspace $\left(X, d_{X}\right)$ of $\mathbb{U}_{1}$ with $d_{X}<1$, define $B\left(X, d_{X}\right)$ as $B\left(S_{Z}\left(X, d_{X}\right), X, d_{X}\right)$.

In principle, the blow-up construction depends only on the enumeration (with repetitions) of pairs of points in $D$. We keep, however, the sequence of $p_{n}$ 's for reference to the further construction. Note that each pair of distinct points in $D$ appears infinitely often in the sequence. Analogous arguments as in Sect. 6, show that the blow-up depends neither on the ordering $p_{n}$ 's nor on the choice of the dense set.

Proposition 7.2 Suppose $S$ and $T$ are simplices, $X \subseteq S$ and $Y \subseteq T$ are their subsets, $d_{X}, d_{Y}<1$ are metrics on $X$ and $Y$, respectively and $D \subseteq X$ and $E \subseteq Y$ are countable dense sets. Given an affine homeomorphism $\varphi: S \rightarrow T$ such that $\varphi^{\prime \prime} X=Y$ and $\varphi \uparrow X$ is an isometry of $\left(X, d_{X}\right)$ and $\left(Y, d_{Y}\right)$, there is an affine homeomorphism $\bar{\varphi}: B\left(S, X, d_{X}\right) \rightarrow B\left(T, Y, d_{Y}\right)$ such that $\bar{\varphi}$ extends $\varphi$ and 
(a) for every $p \in \operatorname{Sch}\left(X, \frac{1}{2} d_{X}, D\right)$ there exists $q \in \operatorname{Sch}\left(Y, \frac{1}{2} d_{Y}, E\right)$ such that if $p=\left(x_{1}, x_{2}, s, t\right)$, then $q=\left(y_{1}, y_{2}, s, t\right)$ and $\bar{\varphi}$ maps $c_{1}(p)$ to $c_{1}(q)$ and $c_{2}(p)$ to $c_{2}(q)$,

(b) for every $q \in \operatorname{Sch}\left(Y, \frac{1}{2} d_{Y}, E\right)$ there exists $p \in \operatorname{Sch}\left(X, \frac{1}{2} d_{X}, D\right)$ such that if $q=\left(y_{1}, y_{2}, s, t\right)$, then $p=\left(x_{1}, x_{2}, s, t\right)$ and $\bar{\varphi}^{-1}$ maps $c_{1}(q)$ to $c_{1}(p)$ and $c_{2}(q)$ to $c_{2}(p)$.

Proof The proof is essentially the same as that of Proposition 6.6 and we only sketch it. Write $B_{n}$ for the simplices in the inverse system of $B\left(S, X, d_{X}\right)$ and $C_{n}$ for the simplices in the inverse system of $B\left(T, Y, d_{Y}\right)$. By induction on $i \in \mathbb{N}$, construct an approximate intertwining $\varphi_{i}: B_{n_{i}} \rightarrow C_{m_{i}}$ and $\psi_{i}:$ $C_{m_{i}} \rightarrow B_{n_{i+1}}$ so that $\varphi_{0}=\varphi$. At the inductive construction, when extending a map from $B_{n_{i}}$ to $B_{n_{i+1}}$, make sure that if $p_{n}(D)=\left(x_{1}, x_{2}, s, t\right)$, then for some $m \in \mathbb{N}$ with $p_{m}(E)=\left(y_{1}, y_{2}, s, t\right)$, the point $c_{1}\left(p_{n}(D)\right)$ is mapped to $c_{1}\left(p_{m}(E)\right), c_{2}\left(p_{n}(D)\right)$ is mapped to $c_{2}\left(p_{m}(E)\right)$ so that

$$
d_{T}\left(\varphi\left(x_{1}\right), y_{1}\right), d_{T}\left(\varphi\left(x_{2}\right), y_{2}\right)<2^{-m}
$$

and $d_{Y}\left(y_{1}, y_{2}\right) \in(s, t)$. Analogous conditions apply when we extend a map from $C_{m_{i}}$ to $C_{m_{i+1}}$. The fact that the above is possible follows at once from the fact that $\varphi$ was an isometry and the elements in the schemes are enumerated with infinite repetitions.

Once the construction is finished, (4) and the symmetric condition involving $d_{S}$ imply that the sequence forms an approximate intertwining. Then, an application of Proposition 4.1 gives an affine homeomorphism $\bar{\varphi}: \lim B_{i} \rightarrow \lim C_{i}$. The conditions (a) and (b) follow directly from the construction.

\section{Coding a metric into the affine structure of a simpliex}

Given a metric space $\left(X, d_{X}\right)$ with $d_{X}<1$, which we assume is a subspace of the Urysohn sphere $\mathbb{U}_{1}$, and a dense countable subset $D$ of $X$, let $\left(p_{n}=\left(\left(x_{n}, y_{n}, s_{n}, t_{n}\right): n \in \mathbb{N}\right)\right.$ be the associated metric scheme of $\left(X, \frac{1}{2} d_{X}\right)$. Consider the blow-up $B\left(X, d_{X}\right)$ and let $M\left(X, d_{X}, D\right)$ be the subset of $B\left(X, d_{X}\right)$ consisting of the points

$$
q c_{1}\left(p_{n}\right)+(1-q) c_{2}\left(p_{n}\right) \text { and } q c_{2}\left(p_{n}\right)+(1-q) c_{1}\left(p_{n}\right)
$$

for $q \in\left(s_{n}, t_{n}\right) \cap \mathbb{Q}$ (note that the points $c_{1}\left(p_{n}\right)$ and $c_{2}\left(p_{n}\right)$ do not belong to the closure of this set since $\left(s_{n}, t_{n}\right) \cap \mathbb{Q}$ is isolated away from 0$)$.

Use the Kuratowski-Ryll-Nardzewski theorem [46, Theorem 12.13], to find a countable dense subset $D(X)$ for every (nonempty) closed subset $X$ of $\mathbb{U}_{1}$ so that the map $D: X \mapsto D(X)$ is Borel. Define now $\Phi\left(X, d_{X}\right)$ as cone $\left(B\left(X, d_{X}\right), M\left(X, d_{X}, D(X)\right)\right.$. It is easy to check that $\Phi$ is a Borel map 
from the space of closed (nonempty) subsets of $\mathbb{U}_{1}$ to the space of separable Choquet simplices (cf. Sect. 2 for the Borel structure on the space of simplices). In fact, the map $(X, D) \mapsto \operatorname{cone}\left(B\left(X, d_{X}\right), M\left(X, d_{X}, D\right)\right)$, which maps a pair of a Polish space and its dense countable subset to a simplex, is continuous in the following natural topologies. First, any such pair $(X, D)$ with $X \subseteq \mathbb{U}_{1}$ is uniquely determined by $D$ (as $X=\bar{D}$ ) and hence the topology on such pairs can be taken as the topology on the space on sequences of elements of $\mathbb{U}_{1}$, which is the product topology on $\left(\mathbb{U}_{1}\right)^{\mathbb{N}}$. Second, the simplices cone $\left(B\left(X, d_{X}\right), M\left(X, d_{X}, D\right)\right)$ obtained from the pairs $(X, D)$ are naturally embedded into the Hilbert cube $[0,1]^{\mathbb{N}}$, and the natural topology there is the Vietoris topology on compact subsets of the Hilbert cube.

Proposition 8.1 Suppose $\left(X, d_{X}\right)$ and $\left(Y, d_{Y}\right)$ are subspaces of $\mathbb{U}_{1}$ with $d_{X}, d_{Y}<1$. If $\left(X, d_{X}\right)$ and $\left(Y, d_{Y}\right)$ are isometric, then the simplices $\Phi\left(X, d_{X}\right)$ and $\Phi\left(Y, d_{Y}\right)$ are affinely homeomorphic.

Proof For simplicity, identify $X$ with $Z(X)$ and $Y$ with $Z(Y)$ (see Proposition 3.2) and let $\varphi: \mathbb{U}_{1} \rightarrow \mathbb{U}_{1}$ be an isometry such that $\varphi^{\prime \prime} X=Y$. By Propositions 5.21 and 7.2, there is an affine homeomorphism $\bar{\varphi}: B\left(X, d_{X}\right) \rightarrow$ $B\left(Y, d_{Y}\right)$ which extends $\varphi$ and such that

- for each $p=\left(x_{1}, x_{2}, s, t\right)$ in the scheme of $\left(X, \frac{1}{2} d_{X}\right)$ there is $q=$ $\left(y_{1}, y_{2}, s, t\right)$ in the scheme of $\left(Y, \frac{1}{2} d_{Y}\right)$ such that $\bar{\varphi}$ maps the cone points $c_{1}(p)$ to $c_{1}(q)$ and $c_{2}(p)$ to $c_{2}(q)$,

- for each $q=\left(y_{1}, y_{2}, s, t\right)$ in the scheme of $\left(Y, \frac{1}{2} d_{Y}\right)$ there is $p=$ $\left(x_{1}, x_{2}, s, t\right)$ in the scheme of $\left(X, \frac{1}{2} d_{X}\right)$ such that $\bar{\varphi}^{-1}$ maps $c_{1}(q)$ to $c_{1}(p)$ and $c_{2}(q)$ to $c_{2}(p)$.

The above imply that $\bar{\varphi}^{\prime \prime} \operatorname{cl}\left(M\left(X, d_{X}, D(X)\right)\right)=\operatorname{cl}\left(M\left(Y, d_{Y}, D(Y)\right)\right)$ and hence, by Proposition 6.6, there is an affine homeomorphism $\overline{\bar{\varphi}}: \Phi\left(X, d_{X}\right) \rightarrow$ $\Phi\left(Y, d_{Y}\right)$ that extends $\bar{\varphi}$.

Proposition 8.1 shows that $\Phi$ maps isometric metric spaces to affinely homeomorphic simplices. To show that $\Phi$ is a reduction, we will restrict attention to perfect separable metric spaces.

Claim 8.2 If $\left(X, d_{X}\right)$ is perfect with $d_{X}<1$, then the set of nonisolated extreme points of $\Phi\left(X, d_{X}\right)$ is equal to $\operatorname{ext}\left(S_{Z}\left(X, d_{X}\right)\right)$.

Proof $X$ is perfect and dense in $\operatorname{ext}\left(S_{Z}\left(X, d_{X}\right)\right)$ by Corollary 5.20, so all extreme points of $S_{Z}\left(X, d_{X}\right)$ are nonisolated in $\operatorname{ext}\left(S_{Z}\left(X, d_{X}\right)\right)$. Since the simplex $S_{Z}\left(X, d_{X}\right)$ is a face of $\Phi\left(X, d_{X}\right)$, the extreme points of $S_{Z}\left(X, d_{X}\right)$ are still nonisolated extreme points of $\Phi\left(X, d_{X}\right)$. Now, Lemmas 6.9 and 6.10 and analogous statements for the blow-up (here we use the fact that $[s, t] \subseteq\left(0, \frac{1}{2}\right)$ for $s<t$ appearing in the metric scheme of $\left(X, \frac{1}{2} d_{X}\right)$ in order to ensure that 
$c_{1}\left(p_{n}\right)$ and $c_{2}\left(p_{n}\right)$ remain isolated after passing from $B\left(X, d_{X}\right)$ to $\left.\Phi\left(X, d_{X}\right)\right)$ imply that all the other extreme points of $\Phi\left(X, d_{X}\right)$ are isolated.

Lemma 8.3 Suppose $\left(X, d_{X}\right)$ is a perfect metric subspace of $\mathbb{U}_{1}$. If $x, y \in X$ and $x \neq y$, then $\frac{1}{2} d_{X}(x, y)$ is the only $\alpha \in\left(0, \frac{1}{2}\right)$ such that

$$
\begin{aligned}
& \forall s<t \in \mathbb{Q} \cap\left(0, \frac{1}{2}\right) \text { with } \alpha \in(s, t) \\
& \exists V_{x}, V_{y} \subseteq \Phi\left(X, d_{X}\right) \text { open neighborhoods of } x \text { and } y \text { in } \Phi\left(X, d_{X}\right) \\
& \forall c_{1} \in V_{x} \forall c_{2} \in V_{y} \text { isolated extreme points } \\
& \quad\left[\left(\exists \lambda \in\left(0, \frac{1}{2}\right) \lambda c_{1}+(1-\lambda) c_{2}\right.\right.
\end{aligned}
$$$$
\text { is a limit of isolated extreme points of } \left.\Phi\left(X, d_{X}\right)\right)
$$$$
\Rightarrow\left(\exists \lambda \in(s, t) \quad \lambda c_{1}+(1-\lambda) c_{2}\right.
$$$$
\text { is a limit of isolated extreme points of } \left.\left.\Phi\left(X, d_{X}\right)\right)\right] \text {. }
$$

Proof Write $D$ for the countable dense subset $D(X)$ of $X$. By Claim 8.2 and Lemma 6.9, the only isolated extreme points of $\Phi\left(X, d_{X}\right)$ are in the sets $E_{1}=$ $\left\{c_{1}(p), c_{2}(p): p \in \operatorname{Sch}\left(X, \frac{1}{2} d_{X}, D\right)\right\}$ and $E_{2}=\left\{c(z): z \in M\left(X, d_{X}, D\right)\right\}$. It follows from the iterated cone construction that if $z \in M\left(X, d_{X}, D\right)$, then for any isolated extreme point $e$ of $\Phi\left(X, d_{X}\right)$, no point in the set $\{\lambda c(z)+(1-\lambda) e$ : $\lambda \in[0,1]\}$ is a limit of isolated extreme points of $\Phi\left(X, d_{X}\right)$. On the other hand, if $e_{1}, e_{2} \in E_{1}$ are such that for some $\lambda \in[0,1]$ the point $\lambda e_{1}+(1-\lambda) e_{2}$ is a limit of isolated extreme points of $\Phi\left(X, d_{X}\right)$, then there is $p \in \operatorname{Sch}\left(X, \frac{1}{2} d_{X}, D\right)$ such that $\left\{e_{1}, e_{2}\right\}=\left\{c_{1}(p), c_{2}(p)\right\}$. The latter follows from the fact that the intersection of $B\left(X, d_{X}\right)$ with the closure of the set $\left\{c(z): z \in M\left(X, d_{X}, D\right)\right\}$ is exactly the closure of $M\left(X, d_{X}, D\right)$.

Pick $x \neq y \in X$. We claim that $\alpha=\frac{1}{2} d_{X}(x, y)$ satisfies the condition (5). Pick any $(s, t) \subseteq\left(0, \frac{1}{2}\right)$ basic open neighborhood of $\alpha$ and let $\varepsilon>0$ be such that $(\alpha-\varepsilon, \alpha+\varepsilon) \subseteq(s, t)$. Since $X$ is a topological subspace of $S\left(X, d_{X}\right)$, there are $V_{x}^{1}$ and $V_{y}^{1}$ open neighborhoods of $x$ and $y$ in $S\left(X, d_{X}\right)$, respectively, such that $V_{x}^{1} \cap X \subseteq \operatorname{ball}_{\left(X, d_{X}\right)}(x, \varepsilon)$ and $V_{y}^{1} \cap X \subseteq \operatorname{ball}_{\left(X, d_{X}\right)}(y, \varepsilon)$. Let $V_{x}$ and $V_{y}$ be open neighborhoods of $x$ and $y$ in $\Phi\left(X, d_{X}\right)$ such that if $c_{1} \in V_{x}$ and $c_{2} \in V_{y}$, then $\pi\left(c_{1}\right) \in V_{x}^{1}$ and $\pi\left(c_{2}\right) \in V_{y}^{1}$, where $\pi: \Phi\left(X, d_{X}\right) \rightarrow S\left(X, d_{x}\right)$ denotes the projection map. We claim that $V_{x}$ and $V_{y}$ are as needed. Let $c_{1} \in V_{x}$ and $c_{2} \in V_{y}$ be arbitrary extreme isolated points such that for some $\lambda \in\left(0, \frac{1}{2}\right)$ the point $\lambda c_{1}+(1-\lambda) c_{2}$ is a limit of isolated extreme points of $\Phi\left(X, d_{X}\right)$. By the remarks in the previous paragraph, there is $p \in \operatorname{Sch}\left(X, \frac{1}{2} d_{X}, D\right)$ such that $p=\left(d_{1}, d_{2}, q, r\right)$ for some $d_{1}, d_{2} \in D$ with $\left\{\pi\left(c_{1}\right), \pi\left(c_{2}\right)\right\}=\left\{d_{1}, d_{2}\right\}$ and $(q, r) \subseteq\left(0, \frac{1}{2}\right)$ is a basic open neighborhood of $\frac{1}{2} d_{X}\left(d_{1}, d_{2}\right)$. Now, since $c_{1} \in V_{x}$ and $c_{2} \in V_{y}$ we have that $\frac{1}{2} d_{X}\left(d_{1}, d_{2}\right) \in(\alpha-\varepsilon, \alpha+\varepsilon) \subseteq(s, t)$, 
so $(q, r) \cap(s, t)$ is nonempty. Pick any $\lambda \in(q, r) \cap(s, t)$ and note that in either case: $\left(d_{1}, d_{2}\right)=\left(\pi\left(c_{1}\right), \pi\left(c_{2}\right)\right)$ or $\left(d_{1}, d_{2}\right)=\left(\pi\left(c_{2}\right), \pi\left(c_{1}\right)\right)$, the point $\lambda c_{1}+(1-\lambda) c_{2}$ is also a limit of isolated extreme points of $\Phi\left(X, d_{X}\right)$ by the definition of $M\left(X, d_{X}, D\right)$ and the fact that $\lambda \in(q, r)$.

We also need to show that $\alpha$ is the only number is $\left(0, \frac{1}{2}\right)$ satisfying (5) for $x$ and $y$. Pick any $\beta \in\left(0, \frac{1}{2}\right)$ distinct from $\alpha$. Let $\varepsilon>0$ be smaller than $|\alpha-\beta|$. Pick a basic open neighborhood $(s, t) \subseteq(\beta-\varepsilon / 2, \beta+\varepsilon / 2)$ of $\beta$. We claim that $(s, t)$ witnesses that (5) is not satisfied. Let $V_{x}$ and $V_{y}$ be arbitrary open neighborhoods of $x$ and $y$, respectively, in $\Phi\left(X, d_{X}\right)$. Pick $d_{1}, d_{2} \in D$ such that $d_{X}\left(d_{1}, x\right)<\varepsilon / 2, d_{X}\left(d_{2}, y\right)<\varepsilon / 2$ and $d_{1} \in V_{x}, d_{2} \in V_{y}$. Note that $\frac{1}{2} d_{X}\left(d_{1}, d_{2}\right) \in(\alpha-\varepsilon / 2, \alpha+\varepsilon / 2)$ and that $(s, t)$ is disjoint from $(\alpha-\varepsilon / 2, \alpha+\varepsilon /$ $2)$. Find a basic open neighborhood $(q, r)$ of $\frac{1}{2} d_{X}\left(d_{1}, d_{2}\right)$ in $\left(0, \frac{1}{2}\right)$ such that $[q, r] \cap(s, t)=\emptyset$. Find $n \in \mathbb{N}$ big enough so that $p_{n} \in \operatorname{Sch}\left(X, \frac{1}{2} d_{X}, D\right)$ is equal to $\left(d_{1}, d_{2}, q, r\right)$ and such that letting $c_{1}=c_{1}\left(p_{n}\right), c_{2}=c_{2}\left(p_{n}\right)$ we have $c_{1} \in V_{x}$ and $c_{2} \in V_{y}$. Now, there is $\lambda \in\left(0, \frac{1}{2}\right)$ such that $\lambda c_{1}+(1-\lambda) c_{2}$ is a limit of isolated extreme points of $\Phi\left(X, d_{X}\right)$ but the set of such $\lambda$ is equal to $[q, r]$, which is disjoint from $(s, t)$. This shows that $\beta$ does not satisfy $(5)$ and ends the proof.

Now we are ready to finish the proof that $\Phi$ is a reduction.

Proposition 8.4 If $\left(X, d_{X}\right)$ and $\left(Y, d_{Y}\right)$ are perfect closed subspaces of $\mathbb{U}_{1}$ with $d_{X}, d_{Y}<1$ and $\Phi\left(X, d_{X}\right)$ is affinely homeomorphic to $\Phi\left(Y, d_{Y}\right)$, then $\left(X, d_{X}\right)$ is isometric to $\left(Y, d_{Y}\right)$.

Proof Let $\varphi: \Phi\left(X, d_{X}\right) \rightarrow \Phi\left(Y, d_{Y}\right)$ be an affine homeomorphism. Note that $\varphi$ maps nonisolated extreme points of $\Phi\left(X, d_{X}\right)$ to nonisolated extreme points of $\Phi\left(Y, d_{Y}\right)$, so, by Claim 8.2, $\varphi^{\prime \prime} \operatorname{ext}\left(S\left(X, d_{X}\right)\right)=\operatorname{ext}\left(S\left(Y, d_{Y}\right)\right)$. Now, $X \subseteq S\left(X, d_{X}\right)$ and $Y \subseteq S\left(Y, d_{Y}\right)$ are dense $G_{\delta}$ sets, so there is a comeager set $X^{\prime} \subseteq X$ such that $Y^{\prime}=\varphi^{\prime \prime} X^{\prime}$ is comeager in $Y$. Note that since $\varphi$ preserves the topological and affine structure, (5) is preserved by $\varphi$ and so Lemma 8.3 implies that for $x_{1}, x_{2} \in X^{\prime}$ we have $d_{Y}\left(\varphi\left(x_{1}\right), \varphi\left(x_{2}\right)=d_{X}\left(x_{1}, x_{2}\right)\right.$. This means that $\left(X^{\prime}, d_{X}\right)$ and $\left(Y^{\prime}, d_{Y}\right)$ are isometric. Since $X^{\prime}$ is comeager in $X$ and $Y^{\prime}$ is comeager in $Y$, the spaces $X$ and $Y$ are isometric as well.

Theorem 1.4 now follows from Propositions 3.1, 8.1 and 8.4.

\section{Concluding remarks and open questions}

As we have mentioned in the introduction, the theory of functorial reductions is still to be developed in the context of descriptive set-theoretic complexity theory. One way to make it precise can be made in terms of Polish groupoids. In this setting, a functorial reduction would be a full (Borel) functor between 
Polish groupoids. It is not difficult to check that our reduction of the isometry of complete separable metric spaces to the affine homeomorphism of Choquet simplices is in fact functorial in the above sense. Another notion of functoriality can be also defined in terms of functors on small categories in order to capture all morphisms between objects. This setting is more subtle.

Another major problem that is still left open is that of the complexity of the homeomorphism relation for compact subspaces of $[0,1]^{2}$. It is known that the homeomorphism relation for compact subspaces of the interval is classifiable by countable structures but the homeomorphism relation in two dimensions is turbulent as shown by Hjorth [40].

Question 9.1 Is the relation of homeomorphism of compact subsets of $[0,1]^{2}$ (or two-dimensional compact spaces) a complete orbit equivalence relation?

In [28, Theorem 7.3] Farah, Toms and Törnquist showed that the isomorphism of separable simple nuclear $\mathrm{C}^{*}$-algebras is below an action of the automorphism group of the Cuntz algebra $\operatorname{Aut}\left(\mathcal{O}_{2}\right)$. Since complete orbit equivalence relations are typically induced by actions of universal Polish groups, the following question is natural.

Question 9.2 Is the group $\operatorname{Aut}\left(\mathrm{O}_{2}\right)$ a universal Polish group?

On the other hand, the following seems to be unknown:

Question 9.3 Can the group $U(H)$ (the unitary group of the infinitedimensional separable Hilbert space) induce a complete orbit equivalence relation?

Finally, this paper reveals an interesting connection between three categories of objects: separable metric spaces, metrizable Choquet simplices and separable Banach spaces. It is somewhat parallel to the connection between metric spaces and Banach spaces given by the Arens-Eells extensions. Recall that given a separable metric space, its Arens-Eells extension is a separable Banach space and the assignment of the Arens-Eells extensions is isometry invariant. On the other hand, given a simplex, one can look at the space of affine continuous functions on it. Now, the $S$-extensions give an invariant assignment of separable Banach spaces to separable metric spaces that factors through simplices as follows:

metric space $\stackrel{S \text {-extension }}{\longrightarrow}$ Choquet simplex $\stackrel{\text { affine space }}{\longrightarrow}$ Banach space

The three categories of separable metric spaces, metrizable Choquet simplices and separable Banach spaces contain universal objects that have been studied independently. While the universal objects in the first two classes are the 
Urysohn space and the Poulsen simplex, the category of separable Banach spaces contains the Gurariu space [37], which is a separable Banach space with an almost isometric extension property. This space turns out to be unique [57] (see also [51] for a recent proof of this result). It is known that the Gurarii space is isomorphic to the space of affine functions on the Poulsen simplex [57] but the relations between the Urysohn space and the Gurarii space or the Poulsen simplex remain unclear. Fonf and Wojtaszczyk [30] showed that the Gurariî space is not isomorphic to the Arens-Eells extension of the Urysohn space. While we think that the $S$-extension of the Urysohn space is not affinely homeomorphic to the Poulsen simplex, we suspect that the compact convex set $S^{\prime}\left(\mathbb{U}_{1}\right)$ is the Poulsen simplex (see page 21 for the definition).

Question 9.4 Is $S^{\prime}(X)$ always a simplex for a separable metric space $X$ ? Is $S^{\prime}\left(\mathbb{U}_{1}\right)$ affinely homeomorphic to the Poulsen simplex?

Acknowledgments Part of this work was done during the author's stay at the Fields Institute during the thematic program in the fall 2012. The author is grateful for the hospitality of the Fields Institute and would like to thank Antonio Avilés, George Elliott, Ilijas Farah and Stevo Todorčević for many inspiring discussions. The author would also like to thank the anonymous Referee for a number of useful suggestions that improved the quality of this paper.

Open Access This article is distributed under the terms of the Creative Commons Attribution 4.0 International License (http://creativecommons.org/licenses/by/4.0/), which permits unrestricted use, distribution, and reproduction in any medium, provided you give appropriate credit to the original author(s) and the source, provide a link to the Creative Commons license, and indicate if changes were made.

\section{References}

1. AIM Problem List. available at http://aimpl.org/settheorycstar

2. Adams, S., Kechris, A.S.: Linear algebraic groups and countable Borel equivalence relations. J. Am. Math. Soc. 13(4), 909-943 (2000). (electronic)

3. Alfsen, E.M.: Boundary values for homomorphisms of compact convex sets. Math. Scand. 19, 113-121 (1966)

4. Alfsen, E.M.: On the Dirichlet problem of the Choquet boundary. Acta Math. 120, 149-159 (1968)

5. Alfsen, E.M.: Compact convex sets and boundary integrals. Springer, New York (1971). Ergebnisse der Mathematik und ihrer Grenzgebiete, Band 57

6. Asimow, L., Ellis, A.J.: Convexity theory and its applications in functional analysis, vol. 16. London Mathematical Society Monographs. Academic Press Inc. [Harcourt Brace Jovanovich Publishers], London (1980)

7. Bauer, H.: Šilovscher Rand und Dirichletsches Problem. Ann. Inst. Fourier Grenoble 11, 89-136, XIV (1961)

8. Becker, H., Kechris, A.S.: The descriptive set theory of Polish group actions, vol. 232. London Mathematical Society Lecture Note Series. Cambridge University Press, Cambridge (1996)

9. Bogaty̆ , S.A.: Metrically homogeneous spaces. Uspekhi Mat. Nauk 57(2(344)), 3-22 (2002) 
10. Choquet, G.: Lectures on analysis. Vol. II: Representation theory. In: Marsden, J., Lance, T., Gelbart, S. (eds). W. A. Benjamin, Inc., New York-Amsterdam (1969)

11. Clemens, J.D.: Isometry of Polish metric spaces. Ann. Pure Appl. Logic 163(9), 1196-1209 (2012)

12. Clemens, J.D., Gao, S., Kechris, A.S.: Polish metric spaces: their classification and isometry groups. Bull. Symb. Logic 7(3), 361-375 (2001)

13. Ellis, A.J. (ed.): Facial structure of compact convex sets and applications. Report of NATO Advanced Study Institute. University College of Swansea (1972)

14. Effros, E., Elliott, G., Farah, I., Toms, A.: Descriptive Set Theory and Operator Algebras. available at http://www.birs.ca/workshops/2012/12w5099/report12w5099.pdf

15. Edward, G.: Structure in simplexes. Acta Math. Effros. 117, 103-121 (1967)

16. Elliott, G.A., Farah, I., Paulsen, V., Rosendal, C., Toms, A., Törnquist, A.: The isomorphism relation for separable $C^{*}$-algebras. Mathematical Research Letters (to appear)

17. Elliott, G.A.: On the classification of inductive limits of sequences of semisimple finitedimensional algebras. J. Algebra 38(1), 29-44 (1976)

18. Elliott, George A.: On the classification of $C^{*}$-algebras of real rank zero. J. Reine Angew. Math. 443, 179-219 (1993)

19. Elliott, G.A.: The classification problem for amenable $C^{*}$-algebras. In: Proceedings of the International Congress of Mathematicians, Vol. 1, 2 (Zürich, 1994), pages 922-932, Basel (1995). Birkhäuser

20. Elliott, G.A.: Towards a theory of classification. Adv. Math. 223(1), 30-48 (2010)

21. Elliott, G.A., Gong, G.: On the classification of $C^{*}$-algebras of real rank zero. II. Ann. Math. (2) 144(3), 497-610 (1996)

22. Elliott, G.A., Gong, G., Li, L.: On the classification of simple inductive limit $C^{*}$-algebras. II. The isomorphism theorem. Invent. Math. 168(2), 249-320 (2007)

23. Elliott, G.A., Rørdam, M.: Classification of certain infinite simple $C^{*}$-algebras. II. Comment. Math. Helv. 70(4), 615-638 (1995)

24. Elliott, G.A., Toms, A.S.: Regularity properties in the classification program for separable amenable $C^{*}$-algebras. Bull. Am. Math. Soc. (N.S.) 45(2), 229-245 (2008)

25. Farah, I.: Logic and operator algebras. https://www.youtube.com/watch?v=KjkWAIUlk3s

26. Farah, I.: Some problems about operator algebras with set-theoretic flavor (2008). circulated problem list

27. Farah, I., Toms, A., Törnquist, A.: The descriptive set theory of $C^{*}$-algebra invariants. Int. Math. Res. Not. IMRN 22, 5196-5226 (2013). Appendix with Caleb Eckhardt

28. Farah, I., Toms, A.S., Törnquist, A.: Turbulence, orbit equivalence, and the classification of nuclear $C^{*}$-algebras. J. Reine Angew. Math. 688, 101-146 (2014)

29. Ferenczi, V., Louveau, A., Rosendal, C.: The complexity of classifying separable Banach spaces up to isomorphism. J. Lond. Math. Soc. (2) 79(2), 323-345 (2009)

30. Fonf, V.P., Wojtaszczyk, P.: Properties of the Holmes space. Topol. Appl. 155(14), 16271633 (2008)

31. Foreman, M., Rudolph, D.J., Weiss, B.: The conjugacy problem in ergodic theory. Ann. Math. (2) 173(3), 1529-1586 (2011)

32. Gaboriau, D.: Orbit equivalence and measured group theory. In: Proceedings of the International Congress of Mathematicians. Volume III, pages 1501-1527, New Delhi (2010). Hindustan Book Agency

33. Gao, S.: Invariant descriptive set theory, vol. 293. Pure and Applied Mathematics (Boca Raton). CRC Press, Boca Raton, FL (2009)

34. Gao, Su, Kechris, Alexander S.: On the classification of Polish metric spaces up to isometry. Mem. Am. Math. Soc. 161(766), (2003)

35. Glimm, J.G.: On a certain class of operator algebras. Trans. Am. Math. Soc. 95, 318-340 (1960) 
36. Gromov, M.: Metric structures for Riemannian and non-Riemannian spaces. Modern Birkhäuser Classics. Birkhäuser Boston Inc., Boston, MA, English edition (2007)

37. Gurariı̌, V.I.: Spaces of universal placement, isotropic spaces and a problem of Mazur on rotations of Banach spaces. Sibirsk. Mat. Ž. 7, 1002-1013 (1966)

38. Harrington, L.A., Kechris, A.S., Louveau, A.: A Glimm-Effros dichotomy for Borel equivalence relations. J. Am. Math. Soc. 3(4), 903-928 (1990)

39. Haydon, R.: A new proof that every Polish space is the extreme boundary of a simplex. Bull. Lond. Math. Soc. 7, 97-100 (1975)

40. Hjorth, G.: Classification and orbit equivalence relations, vol. 75. Mathematical Surveys and Monographs. American Mathematical Society, Providence, RI (2000)

41. Hjorth, G.: Borel equivalence relations. In: Handbook of set theory. Vols. 1, 2, 3, pages 297-332. Springer, Dordrecht (2010)

42. Huhunaišvili, G.E.: On a property of Uryson's universal metric space. Dokl. Akad. Nauk SSSR (N.S.) 101, 607-610 (1955)

43. Jellett, F.: Homomorphisms and inverse limits of Choquet simplexes. Math. Z. 103, 219226 (1968)

44. Johnson, W.B., Lindenstrauss, J. (eds.): Handbook of the Geometry of Banach Spaces, vol. I. North-Holland Publishing Co., Amsterdam (2001)

45. Joiner, C.: On Urysohn's universal separable metric space. Fund. Math. 73(1), 51-58 (1971/72)

46. Kechris, A.S.: Classical descriptive set theory, vol. 156. Graduate Texts in Mathematics. Springer, New York (1995)

47. Kechris, A.S.: The descriptive classification of some classes of $C^{*}$-algebras. In Proceedings of the Sixth Asian Logic Conference (Beijing, 1996), pages 121-149. World Sci. Publ., River Edge, NJ (1998)

48. Kechris, A.S., Louveau, A.: The classification of hypersmooth Borel equivalence relations. J. Am. Math. Soc. 10(1), 215-242 (1997)

49. Kirchberg, E., Phillips, N.C.: Embedding of exact $C^{*}$-algebras in the Cuntz algebra $\mathrm{O}_{2}$. J. Reine Angew. Math. 525, 17-53 (2000)

50. Kirchberg, E., Rørdam, M.: Non-simple purely infinite $C^{*}$-algebras. Am. J. Math. 122(3), 637-666 (2000)

51. Solecki, S., Kubiś, W.: A proof of uniqueness of the Gurariŭ space. Israel J. Math. (to appear)

52. Lazar, A.J., Lindenstrauss, J.: Banach spaces whose duals are $L_{1}$ spaces and their representing matrices. Acta Math. 126, 165-193 (1971)

53. Leiderman, A., Pestov, V., Rubin, M., Solecki, S., Uspenskij, V.: Preface [Special issue: Workshop on the Urysohn space]. Topology Appl. 155(14), 1451-1452 (2008). Held at Ben-Gurion University of the Negev, Beer Sheva, May 21-24, 2006

54. Lindenstrauss, J., Olsen, G., Sternfeld, Y.: The Poulsen simplex. Ann. Inst. Fourier (Grenoble) 28(1):vi, 91-114 (1978)

55. Lopez-Abad, J., Todorcevic, S.: Generic Banach spaces and generic simplexes. J. Funct. Anal. 261(2), 300-386 (2011)

56. Louveau, A., Rosendal, C.: Complete analytic equivalence relations. Trans. Am. Math. Soc. 357(12), 4839-4866 (2005). (electronic)

57. Lusky, W.: On separable Lindenstrauss spaces. J. Funct. Anal. 26(2), 103-120 (1977)

58. Melleray, J.: Computing the complexity of the relation of isometry between separable Banach spaces. MLQ Math. Log. Q. 53(2), 128-131 (2007)

59. Melleray, J.: On the geometry of Urysohn's universal metric space. Topol. Appl. 154(2), 384-403 (2007)

60. Melleray, J.: Some geometric and dynamical properties of the Urysohn space. Topol. Appl. 155(14), 1531-1560 (2008) 
61. Pestov, V.: Dynamics of infinite-dimensional groups, vol. 40. University Lecture Series. American Mathematical Society, Providence, RI (2006)

62. Phelps, R.R.: Lectures on Choquet's theorem, vol. 1757. Lecture Notes in Mathematics. Springer-Verlag, Berlin, second edition (2001)

63. Ebbe T.P.: A simplex with dense extreme points. Ann. Inst. Fourier. Grenoble 11, 83-87, XIV (1961)

64. Rørdam, M.: Classification of certain infinite simple $C^{*}$-algebras. J. Funct. Anal. 131(2), 415-458 (1995)

65. Rørdam, M.: A simple $C^{*}$-algebra with a finite and an infinite projection. Acta Math. 191(1), 109-142 (2003)

66. Thomas, S.: On the complexity of the classification problem for torsion-free abelian groups of rank two. Acta Math. 189(2), 287-305 (2002)

67. Thomas, S.: The classification problem for torsion-free abelian groups of finite rank. J. Am. Math. Soc. 16(1), 233-258 (2003)

68. Thomsen, K.: Inductive limits of interval algebras: the tracial state space. Am. J. Math. 116(3), 605-620 (1994)

69. Toms, A.S.: On the classification problem for nuclear $C^{*}$-algebras. Ann. Math. (2) 167(3), 1029-1044 (2008)

70. Urysohn, P.S.: Sur un espace métrique universel. Bull. Sci. Math. 51, 43-64 and 74-96 (1927)

71. Vesterstrøm, J.: On open maps, compact convex sets, and operator algebras. J. Lond. Math. Soc. 2(6), 289-297 (1973)

72. Zielinski, J.: The complexity of the homeomorphism relation between compact metric spaces. arXiv:1409.5523 